\title{
Krise im öffentlichen Raum
}

\section{Formen und Funktionen kleiner Kommunikationsformate zu Covid 19}

\author{
Irmtraud Behr $\cdot$ Frank Liedtke
}

Eingegangen: 28. April 2021 / Angenommen: 12. Juli 2021 / Online publiziert: 28. Oktober 2021 (C) Springer-Verlag GmbH Deutschland, ein Teil von Springer Nature 2021

Zusammenfassung In unserem Beitrag werden zunächst grundsätzliche Überlegungen zur medialen Konstitution der Covid-19-Krise sowie zum Begriff der Krise selbst vorgenommen. Im Anschluss daran wird ein umfangreiches Korpus von Aushängen und anderen Kommunikaten aus vier europäischen Ländern - Frankreich, Deutschland, Österreich und Schweden - unter dem Aspekt ihrer Materialität, Medialität und Lokalität sowie der jeweiligen diskursiven Funktionen analysiert. Unter zeitlichem Aspekt werden drei Phasen der Verwendung von Kommunikaten in der Pandemie angenommen. In grundsätzlicher Perspektive geht es sodann um die Eigenschaft der Arealität, die für öffentliche Kommunikate der untersuchten Art charakteristisch ist. Unter Arealität wird die Reichweite oder der Geltungsbereich der Kommunikate mit den jeweils spezifischen Rezeptions- und Befolgungsbedingungen verstanden. Die von uns verfolgte Hypothese lautet, dass eine Typologie der Kommunikate unter dem Aspekt ihrer Arealität aufgestellt werden kann, wodurch vertiefte Einsichten in ihre sozialen und kommunikativen Funktionen ermöglicht werden.

Schlüsselwörter Krise $\cdot$ Krisenkonstitution · Krisenphasen · COVID-19 · CoronaKommunikate $\cdot$ Materialität · Medialität · Lokalität · Arealität

Dieser Artikel ist Teil der Arbeiten des internationalen Forschungsnetzwerkes »Le genre bref dans l'espace public / Brief genre in public space«, das von der Université Sorbonne Nouvelle (Paris), der Aoyama Gakuin University (Tokyo), der Université Gustave Eiffel und der Université de Pau et des Pays de l'Adour (Pau) in Kooperation mit der Universität Leipzig getragen wird.

Irmtraud Behr $(\bowtie)$

Université Sorbonne Nouvelle - Paris 3, Paris, Frankreich

E-Mail: irmtraud.behr@sorbonne-nouvelle.fr

Frank Liedtke

Universität Leipzig, Leipzig, Deutschland

E-Mail: liedtke@uni-leipzig.de 


\section{Crisis in the Public Sphere}

Forms and Functions of Small Forms of Communication with Respect to Covid 19

Abstract In our contribution, we first make fundamental considerations on the medial constitution of the Covid 19 crisis as well as on the concept of crisis itself. Subsequently, an extensive corpus of notices and other communiqués from four European countries - France, Germany, Austria and Sweden - is analysed under the aspect of their materiality, mediality and locality as well as the respective discursive functions. From a temporal perspective, three phases of the use of communicates in the pandemic are assumed. From a fundamental perspective, we then look at the property of areality, which is characteristic of public communicates of the type studied. Areality is understood as the range or scope of the communicates with their specific conditions of reception and compliance. The hypothesis we are pursuing is that a typology of the communicates can be established under the aspect of their areality, thus enabling deeper insights into their social and communicative functions.

Keywords Crisis - Constitution of Crisis - Covid-19 - Communicates of Corona · Materiality $\cdot$ Mediality $\cdot$ Locality $\cdot$ Areality

\section{Einleitung}

Die Entwicklung der Corona-Pandemie ist als Krise zu werten; dies hat sich im Laufe des Jahres 2020 als allgemeine Einschätzung durchgesetzt. Nimmt man die Erwähnung des Wortes Krise im öffentlichen Sprachgebrauch zum Maßstab, so lässt sich dieser Befund für die Printmedien klar bestätigen. Das Zeitungskorpus des DWDS (Digitales Wörterbuch der deutschen Sprache) zeigt, dass die Erwähnung von Krise in deutschsprachigen Zeitungen im Jahre 2020 mit 15.202 Token das

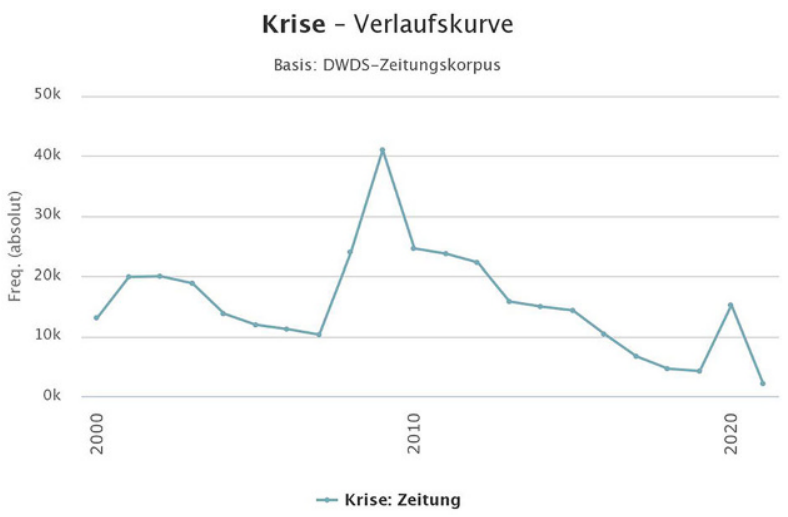

Abb. 1 DWDS-Wortverlaufskurve »Krise« (www.dwds.de, abgerufen am 02.08.2021). DWDS-Wortverlaufskurve für »Krise«, erstellt durch das Digitale Wörterbuch der deutschen Sprache. Die ermittelten Frequenzen betragen jahrgangsweise: 2015: 14.303; 2016: 10.346; 2017: 6.635; 2018: 4573; 2019: 4169; 2020: 15.202 
Niveau von 2015 übertrifft, dem Höhepunkt des Flüchtlingszustroms mit 14.303 Token (s. Abb. 1).

Ein Reflex dieser Entwicklung ist ein Beschluss der Gesellschaft für Deutsche Sprache: Am 30. November wählte sie das Wort Corona-Pandemie zum Wort des Jahres, das in Wortbildungen auch mit dem Kompositum >-krise< vorkommt. In ihrer Begründung führt sie Folgendes aus:

Als Wort des Jahres steht Corona-Pandemie nicht nur für die nach Einschätzung der Bundeskanzlerin ebenso wie vieler Fachleute schwerste Krise seit dem 2. Weltkrieg, sondern sprachlich auch für eine Vielzahl neuer Wortbildungen (Coronavirus, -krise, -zahlen, -jahr, Corona-Demo, -Hotspot, -Warn-App, coronabedingt, -geplagt ...). https://gfds.de/wort-des-jahres-2020-1; abgerufen am 25.02.2021

Angesichts der Präsenz des Krisenbegriffs im öffentlichen Sprachgebrauch sind die Krisenleugner mittlerweile ins Hintertreffen geraten, was eine Erklärung für ihre wachsende Radikalisierung sein mag. Indessen scheint es weniger klar zu sein, wovon man genau spricht, wenn von Krise die Rede ist. Was sind die ihr zugeschriebenen, charakteristischen Eigenschaften, wie lassen sie sich von nicht-krisenhaften Verläufen potenzieller Krisenereignisse unterscheiden? Diese Grundsatzfrage soll am Beginn der folgenden Ausführungen stehen. Der Aspekt der öffentlichen Kommunikation, wie sie sich unter anderem in öffentlichen Aushängen und anderen Kommunikationsformaten spiegelt, bildet im Anschluss daran die zentrale Fragestellung, die in diesem Beitrag behandelt werden soll. Um der Vielfalt dieser Formate gerecht zu werden, verwenden wir den Begriff des Kommunikats als zusammenfassende Bezeichnung für Aushänge, Aufkleber, gesprayte Markierungen, elektronische Anzeigetafeln und Aufsteller.

Leitend für den hier vorgestellten Ansatz sind zwei Annahmen, für die anhand des präsentierten Materials argumentiert werden soll: Indem die untersuchten Kommunikate sich auf Teilphänomene der Krise beziehen, konstituieren sie diese sprachlichdiskursiv in entscheidender Weise mit. Diese konstitutive Funktion soll anhand der Materialität, der Medialität und der Lokalität der Corona-Kommunikate nachgewiesen werden. Die Kommunikate haben dabei nicht nur informative Funktion, sondern offenkundig aufgrund ihres vorschreibenden Charakters auch regulative Funktion; schließlich sind, vor allem zu Beginn der Pandemie im Frühjahr 2020, Kommunikate mit expressiver Funktion häufig.

Die zweite hier zu begründende Annahme bezieht sich auf den Stellenwert dessen, was wir die Arealität der Kommunikate nennen. Der spezifische Ort der Kommunikate, die jeweilige Reichweite ihrer Botschaften, sowie die areale Beziehung zwischen den Kommunikaten und den Rezipient*innen, von denen teilweise ein sehr spezifisches und auf den Moment der Lektüre bezogenes Verhalten erfordert wird, spielen eine entscheidende Rolle bei der Beschreibung der Funktionsweise der Kommunikate. Konstitutive Funktion und Arealität sind somit zentrale Eigenschaften der untersuchten Kommunikate, wobei es sicher lohnend wäre, diese Eigenschaften auch in anderen Fällen öffentlicher Kommunikation mittels kurzer Formate zu untersuchen. 
Unsere Überlegungen stützen sich auf Beobachtungen in Deutschland, Frankreich, Österreich und Schweden zwischen März 2020 und März 2021. Die Kommunikate sind fotografisch dokumentiert, datiert und lokalisiert. ${ }^{1}$

\section{Diskursive Krisenkonstitution}

Die sprunghaft angestiegenen Zahlen von Zeitungsmeldungen, wie sie sich in der Zahl der gemessenen Token des Wortes Krise für das Jahr 2020 spiegeln, aber auch öffentliche Aushänge, die vielfältigen Ver- und Gebote, die medial weit verbreiteten Aufrufe und motivierenden Durchhalteappelle sind Teil dessen, was als eine diskursiv-sprachliche Fassung und damit auch eine gesellschaftliche Konzeptualisierung der Krise gelten kann. Öffentliche Kommunikationsformate und ihre Erscheinungsformen beziehen sich auf einzelne Aspekte der krisenhaften Ereignisse, und sie sind gleichzeitig selbst Teil der Krise - ja der >unsichtbare Feind < des Infektionsgeschehens wird durch diese zum Teil erst sichtbar gemacht. Eine Zeitungsmeldung, dass die Zahl der Infektionen wieder gestiegen ist, oder auch ein Aushang an einer Schaufensterscheibe, dass dieses bestimmte Geschäft aufgrund der Corona-Krise geschlossen ist, informieren über die Entwicklung der Pandemie oder über die Schließung des Geschäfts, wobei offenkundig der Bericht oder die Information nicht nur von der Krise oder ihren Folgen handelt, sondern sie auch mitkonstituiert.

Zunächst jedoch zum Grundsätzlichen: Wovon sprechen wir, wenn wir von Krise sprechen? Ursprünglich als ein Zeitpunkt im Krankheitsverlauf verstanden, an dem sich der gute oder schlechte Ausgang ihrer Entwicklung entscheidet, hat sich der Begriff aus dem medizinischen Kontext herausentwickelt und wurde entscheidend verallgemeinert. Krise wird als ein Herausfallen aus einer Normalität oder einer Routine gefasst, wobei dieses Herausfallen Risiken in sich birgt, aber auch Möglichkeiten der Innovation und der Weiterentwicklung. Für den Soziologen Ulrich Oevermann ist das Spannungsverhältnis von Krise und Routine entscheidend, wobei sich in bestimmten gesellschaftlichen oder historischen Konstellationen entscheidet, »ob etwas als Krise thematisch ist oder in einer Routine für gültig gehalten wird « (Oevermann 2006, S. 85). In einer wissenssoziologischen Sicht geht Oevermann von der Struktur einer Proposition aus, die einen Referenzausdruck X und ein Prädikat $P$ enthält und in Gänze somit > $X$ ist $P$ < ergibt. Das individuelle $X$ wird dann in einem Erkenntnisakt dem allgemeinen $\mathrm{P}$ zugewiesen. Dies heißt für das Verhältnis von Krise und Routine: »Die Realität der >X.e<, also die Wirklichkeit, steht zugleich für die Krise, und die Realität des Begriffs-Allgemeinen, der Prädikate, für die Routine « (ebd., S. 91). Sobald ein Gegenstand auftaucht, der noch nicht einem Allgemeinen zugeordnet werden kann, ergibt sich eine für die Erkenntnis krisenhafte Situati-

\footnotetext{
1 Die Auswahl der Länder lässt sich auf Aufenthalte der Verf. in dem fraglichen Zeitraum der Jahre 2020-2021 zurückführen. S. auch Androutsopoulos 2020, der die Anfangsphase der Krise in Hamburg beobachtet. Ein kollaboratives Projekt VintrinesEnConfinement (https://vitrinesenconfinement.huma-num. fr/, 4.4.2021) wird von Sarah Gensburger (ISP/CNRS) \& Marta Severo (Dicen-IDF/Université Paris Nanterre) getragen, das vorzüglich den ersten Lockdown dokumentiert.
} 
on, die erst dann >geheilt< ist, wenn eine solche Zuordnung zu einem allgemeinen Prädikat geleistet ist. ${ }^{2}$

Die von Oevermann vorgenommene Strategie, die wir hier nur verkürzt wiedergeben konnten, verlegt den krisenlösenden Zuordnungsprozess von Einzelgegenständen zu allgemeinen Prädikaten in die Struktur einer Proposition. Propositionen sind Bestandteile von Sprechakten, und so unterliegt diesem Modell die Annahme, dass das Verhältnis von Krise und Routine in den propositionalen Gehalt eines Sprechakts >eingebaut< ist (s. ebd, S. $101 \mathrm{ff}$.). Dagegen kann man einwenden, dass die Struktur einer Proposition, sei es im Rahmen der Sprechakttheorie oder unabhängig davon, etwa im Sinne Russells, nicht von einer Abfolgebeziehung ihrer Teile ausgeht. Sie macht keine Aussagen darüber, ob es >zuerst< ein X gibt, das >dann< einem Prädikat $\mathrm{P}$ zugeordnet wird; $>\mathrm{X}$ ist $\mathrm{P}<$ ist einfach die Struktur, die Propositionen oder propositionalen Gehalten eigen ist, ohne dass diese zeitlichen Beziehungen eine Rolle spielen. Abgesehen von diesem kritischen Einwand ist der Oevermannsche Gedanke, das Verhältnis von Krise und Routine als etwas anzusehen, das Bestandteil eines Sprechakts - in diesem Fall der Behauptung - ist, durchaus produktiv. Er besagt nämlich, dass das Verhältnis von Krise und Routine und auch der Begriff der Krise selbst nicht unabhängig davon bestehen, ob Behauptungen über Krisen aufgestellt werden. Hier findet das eingangs betonte Element der diskursiven Konstituierung seine Entsprechung.

In ihrer Untersuchung über Unsicherheit, Krise und Routine schließt Aida Bosch an den Oevermannschen Ansatz an. Sie versteht unter Krise »zugespitzte Situationen, in denen Routinen sowie eingespielte Handlungsformen oder institutionelle Lösungen nicht mehr die gewünschten Ergebnisse bringen, sie funktionieren nicht mehr« (Bosch 2015, S. 213). Die Rolle von Routinen sieht sie darin, dass sie die Wahrnehmung und das Handeln entlasten und somit erst die Möglichkeit eröffnen, auf Krisen zu reagieren. Krisen haben einerseits bedrohlichen Charakter, ermöglichen andererseits erst einen bestimmten Wandel und eine Weiterentwicklung auch von Routinen: »Routinen schließen die Wahrnehmung und das Handeln, Krisen öffnen die Wahrnehmung und damit auch die Handlungsmöglichkeiten.« (ebd. S. 213)

Dieses Spannungsverhältnis von Krise und Routine ist bei Oevermann darin angelegt, dass der krisenhafte Referenzakt ja auch eine Neuentdeckung sein kann, die dann durch Zuordnung zu einem Prädikat verallgemeinert wird. Diese Konstellation prägt sicher nicht nur die Krisenverläufe im individuellen Erkenntnisakt oder in einer individuellen Handlungssituation, wie sie von Bosch (2015) thematisiert wurde, sondern auch in größerem Maßstab in Bezug auf eine Gesellschaft oder, wie im Falle der Corona-Pandemie, in Bezug auf eine Art Weltgesellschaft. Hier können möglicherweise auf technischer Ebene die schnelle Entwicklung von Impfstoffen und ihre Distribution, auf politisch-sozialer Ebene z.B. die Entwicklung neuer Solidaritätspraktiken genannt werden.

\footnotetext{
2 Z.B. stellt die plötzliche Unmöglichkeit, im Restaurant zu essen, einen Routinebruch zum Zeitpunkt $\mathrm{t}_{\mathrm{n}}$ dar « $\neg(\text { Im-Restaurant-essen-können= Routine })_{\mathrm{tn}} »$. Die Ausweichmöglichkeit, Essen im Restaurant zu bestellen, wird nach einer Zeit der Gewöhnung zum Zeitpunkt $t_{n+1}$ eine neue Routine: «(Essen-im-Restaurant-bestellen $=$ Routine $)_{\mathrm{tn}+1}{ }^{»}$.
} 
In der Perspektive der kritischen Diskursanalyse hebt David Römer den schon angesprochenen konstitutiven Aspekt der medialen Krisenkommunikation hervor, indem er festhält: »Eine Krise erhält ihre spezifische Qualität allerdings erst, wenn sie diskursiv-sprachlich repräsentiert wird « (Römer 2020, S. 233). Diese Repräsentation ist immer schon interessegeleitet, denn »ein zentraler Aspekt von Krisendiskursen sind [...] immer auch Deutungskämpfe, etwa um die richtigen Strategien zur Krisen-Bewältigung « (ebd.; s. hierzu auch Wengeler/Ziem 2010). Im Rahmen der kritischen Diskursanalyse (s.u.a. Parr 2013) spielt dabei der Begriff der Kollektivsymbole eine wichtige Rolle, die »bestimmte Deutungen nahelegen, Komplexität reduzieren und (wissenschaftliche) Spezialdiskurse in Beziehung setzen« (ebd., S. 234). Kollektivsymbole gelten somit als »verbindende Elemente innerhalb einer ausdifferenzierten Gesellschaft« (ebd.). Wir werden bei der Analyse der coronaspezifischen Aushänge und anderen Kommunikate sehen, dass dieser Begriff durchaus seine deskriptive Entsprechung in Inhalt und Gestaltung dieser Kommunikate hat. Dies gilt für die Übertragung wissenschaftlicher, virologischer oder statistischer Sachverhalte in konkrete Handlungsanweisungen (z.B. AHA-Regeln/gestes barrières) aber auch für erwünschte kollektive Haltungen und Praktiken wie Solidarität, Durchhaltevermögen und anderes.

\section{Corona-Kommunikate}

\subsection{Stellenwert im Gesamtdispositiv}

Welche Rolle spielen nun die von uns untersuchten kleinen Kommunikationsformate in der gesamten Diskurswelt der Corona-Pandemie? Welchen Stellenwert haben sie im Gesamtdispositiv der Corona-Bewältigung, wer verfasst sie und an wen sind sie gerichtet? Schließlich interessiert die Frage, ob sie an schon vorhandene Diskurstypen anschließen.

Sie sind in ihrer Kürze, ihrer Text-Bild-Kombinatorik und auch im Ort ihrer Präsentation von Zeitungsmeldungen, medial verbreiteten Stellungnahmen und Ansprachen sowie von Podcasts und Ähnlichem deutlich unterschieden. Bei aller medialer Schriftlichkeit weisen sie oftmals konzeptionelle Mündlichkeit auf, wobei diese auf der Seite der Medialität von handschriftlicher Typografie begleitet sein kann. Jannis Androutsopoulos betrachtet diese Formate als einen Gegenstand der linguisticlandscape-Forschung: »Die Sprachlandschaft der Pandemie besteht aus allen sprachlichen Zeichen und ihren Trägerflächen, die Wissen von der Pandemie und von den Praktiken ihrer Bekämpfung aufgreifen und dadurch die Pandemie als öffentlichen Zustand diskursiv konstituieren « (Androutsopoulos 2020, S. 294). ${ }^{3}$ In seinem Ansatz bezieht er sich auf den foucaultschen Begriff des Dispositivs, das »als ein emergentes Geflecht zu begreifen ist, das durch Handlungen unterschiedlicher Akteure entsteht

\footnotetext{
3 Ähnlich steht es mit der Vigipirate-Sprachlandschaft in Frankreich. Als eines der zentralen Instrumente im Anti-Terrorismus-Dispositiv wurde der Plan Vigipirate 2016 aktualisiert. Vigipirate-Kommunikate und Pandemie-Kommunikate können also an manchen Orten gemeinsam auftreten. (https://www. gouvernement.fr/risques/comprendre-le-plan-vigipirate, 4.4.2021).
} 
und Semiotisches, Materielles und Körperliches in eine neue Ordnung einspannt « (ebd., S. 292). Hinzu treten in dem hier untersuchten Material bildhafte Elemente sowie symbolische Markierungen, die etwa die einzuhaltenden Abstände zwischen Personen im öffentlichen Raum markieren. Im erweiterten Sinne handelt es sich somit um Zeichenlandschaften, die die Pandemie anzeigen sowie konstituieren.

Der öffentliche Raum ist grundsätzlich ein Ort, der für Mitglieder einer Gesellschaft durch spezifische Handlungsroutinen gekennzeichnet ist. Diese gelten nicht immer und überall, sondern werden in besonderen Situationen bisweilen durchbrochen. Peter Auer sieht in der öffentlichen Verwendung von Schrift genau hier eine spezifische Funktion: »Die öffentliche Verwendung der Schrift wird dann relevant, wenn der Raum sich nicht ohne Weiteres in (...) allgemein bekannte Orte routinehaften Handelns gliedert, sondern für bestimmte Nutzer erst interpretiert [...] werden muss « (Auer 2010, S. 275). Vor dem Hintergrund der Überlegungen zu Routine und Krise können wir ergänzen, dass die Relevanz für öffentlichen Schriftgebrauch durch den potenziell krisenhaften Charakter der Situationen entsteht, die noch interpretationsbedürftig sind. In dieser Sichtweise geben die coronaspezifischen Aushänge auch eine Orientierung für die Rezipient*innen, wie sie sich an prekären Orten verhalten sollten oder müssen (oder nicht dürfen).

\subsection{Lektüresituation und Lektürekontext}

Die Form der kleinen Kommunikationsformate ist durch diese Funktion bestimmt, denn sie sind stark auf den Lektürekontext zugeschnitten. Anders wäre ihre kurze und in vielen Fällen elliptische Gestalt nicht zu erklären. Für Heiko Hausendorf kommt der Textcharakter der Kommunikate »vor allem aus dem Ausschöpfen der Lektüresituation (kraft sinnlicher Wahrnehmung) und des Lektürekontextes (kraft erworbenen Wissens) zustande « (Hausendorf 2009, S. 8). Die Lektüresituation würde man entsprechend so charakterisieren, dass sie in dem Betreten eines bestimmten Ortes besteht - dies kann ein Geschäft, eine Behörde, eine Kulturinstitution oder eine Bildungsinstitution sein. Bisweilen werden auch definierte Bereiche $>$ unter freiem Himmel< entsprechend reglementiert. Der Lektürekontext, der nach Hausendorf das erworbene beziehungsweise das vom jeweiligen Aushang vorausgesetzte Wissen umfasst, besteht in unserem Fall einerseits in dem ortsspezifischen Routinewissen, andererseits in dem Wissen um das Pandemiegeschehen, also schlichtweg in dem Wissen, dass man sich in einer Corona-Pandemie befindet.

Im Gegensatz zu kommerziellen, aber ähnlich wie politische Kommunikate sind kleinformatige Corona-Kommunikate den Ereignissen und öffentlichen Maßnahmen nachgeschaltet, es sind reaktive Wortmeldungen im allgemeinen Corona-Diskurs oder Transkriptionen der beschlossenen öffentlichen Maßnahmen. Mit Ludwig Jäger kann in diesem Zusammenhang von einer medialen Transkriptivität ausgegangen werden: Dabei wird Transkription aufgefasst als »[...] ein intermediales Verfahren, das Symbolsysteme verschiedener medialer Provenienz so zueinander positioniert, dass sie sich gegenseitig - oder asymmetrisch eines das andere - semantisch erschließen« (Jäger 2010, S. 30f.). Ein Aushang, der über bestimmte Restriktionen informiert oder diese einführt, kann als transkriptiver Verweis auf eine staatliche Verordnung gelesen werden, die diese Maßnahme erst legitimiert. Bisweilen wird 
Abb. 2 Deutschland, 2020, Aushang am Fenster einer Postfiliale. (a) [Sprechblase] (b) BITTE NUR MIT ABSTAND UND GESICHTSMASKE! (c) [Fließtext]

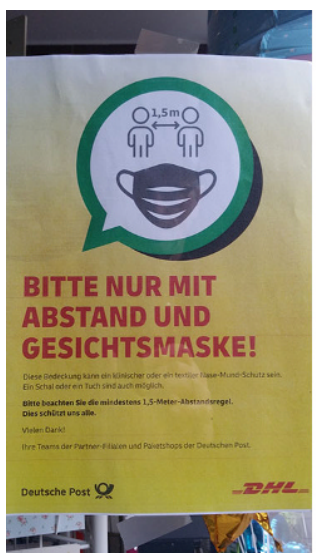

dieser Bezug auch explizit genannt, indem die Verordnungen oder ihre gesetzlichen Grundlagen zitiert werden.

Von wenigen Ausnahmen abgesehen wie z.B. dem verstärkten Einsatz von Bodenmarkierungen, auf die noch zurückzukommen sein wird, nutzen die kleinformatigen Corona-Kommunikate schon bewährte Formen der schriftlichen Kommunikation im öffentlichen Raum, vor allem Plakate und Aushänge verschiedener Größen, teilweise zusammen mit bildlichen Elementen. Wie in der Klassifikation der Kommunikate gezeigt wird, handelt es sich dabei um handgeschriebene oder vorgedruckte, standardisierte, um semiotisch einfache oder multimodale, schnell erkennbare (vgl. Kavanagh et al. 2016) Formate.

Nur durch das Zusammenspiel von Kontextwissen und Lektüre kann somit die Interpretation der teils sehr voraussetzungsreichen Aushänge überhaupt erklärt werden. Sehen wir uns hierfür ein erstes Beispiel (Abb. 2) ${ }^{4}$ an, einen Aushang am Eingang einer Postfiliale.

Auffallend ist hier zunächst die Verblosigkeit des rot gehaltenen Haupttextes (b), der die eigentliche Handlung nicht benennt, sondern als selbstverständlich voraussetzt. Dies entspricht der empraktischen Zeichenverwendung bei Bühler (1934, $\S 10,2)$. Benannt wird nur die Art und Weise, wie das Betreten der Postfiliale erfolgen sollte, wobei diejenigen, zwischen denen der Abstand gehalten werden soll, auch nicht erwähnt werden. Zusätzlich wird der Inhalt der Bitte noch einmal bildhaft in einer Art Sprechblase (a) konkretisiert, wobei die Personen mit dem entsprechenden Abstand von 1,5 Metern ikonisch abgebildet sind. Im fortlaufenden Text (c) wird die ergangene Bitte begründet mit einem für viele Aushänge typischen Motiv, demjenigen des kollektiven Guts. Indem darauf hingewiesen wird, dass die Abstandsregel >uns alle< schützt, wird an die Solidarität jedes Einzelnen appelliert gegenüber der Gemeinschaft, deren Teil er/sie ist. Wir können mit Auer festhalten, dass das routinehafte Betreten einer Postfiliale durch die Erfordernis durchkreuzt wird, dies in einer bestimmten Weise mit einer bestimmten Art der Gesichtsbedeckung zu tun - was nach Oevermann und Bosch ein krisenhaftes Moment im Alltagshandeln darstellt.

\footnotetext{
4 Alle Fotos: (C) ibehr, fliedtke, h.e.karlsson, rbehr.
} 
Mit Hausendorf können wir das Plakat eingewoben sehen in eine bestimmte Lektüresituation - das Betreten einer Filiale - mit einem spezifischen Hintergrundwissen der Coronapandemie, was beides die elliptische Form des Aushangs erklären kann.

Die solidaritätsmarkierende Funktion der Wendung >uns alle $<$ hat dabei zweifellos die Funktion, Zustimmung zu den verhängten Maßnahmen zu erzeugen und gemeinschaftliches Handeln zu erreichen (s. hierzu Spieß 2020, S. 206). Sie findet sich in dieser oder in einer ähnlichen Form, unter Verwendung von Ausdrücken wie >zusammen<, >gemeinsam $<$ oder auch >Sie und Ich $<$, auf vielen Aushängen wieder. ${ }^{5}$ Mit ihnen wird auf eine Akzeptanz abgezielt, die ein eigenverantwortliches Verhalten voraussetzt, was natürlich eine bessere Grundlage für ihre Befolgung darstellt als einfache Ver-/Gebote mit der Androhung von Sanktionen. Dies kann auch als Versuch gelten, die mehr oder minder abstrakte, kollektive Ebene (der Eindämmung der Virusverbreitung durch >Abflachen < der Infektionskurve) und die individuelle Ebene des einzelnen Verhaltens besser miteinander zu vereinbaren (s. hierzu Liedtke 2020).

\section{Materialität, Medialität und Lokalität}

\subsection{Materialität}

Unter dem Aspekt der formalen Gestaltung lassen sich verschiedene Klassen von Kommunikaten bilden. Hier ist zunächst der Gesichtspunkt der Materialität leitend: Sind die Kommunikate als vorgedruckte, gesetzte und anspruchsvoll gestaltete Aus-
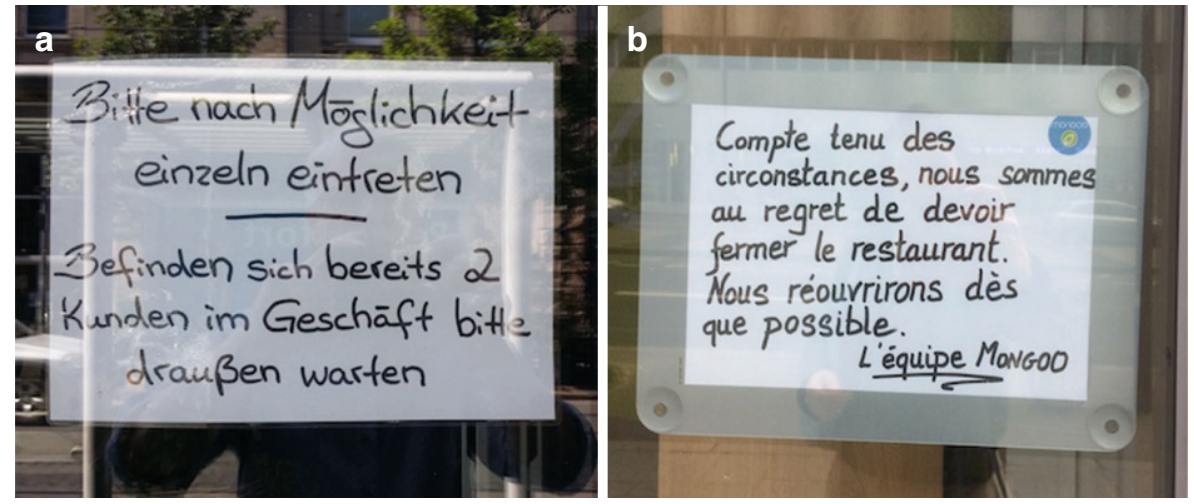

Abb. 3 Aushänge im Schaufenster eines Geschäfts/Restaurants. a Düsseldorf, Frühjahr 2020: Bitte nach Möglichkeit einzeln eintreten Befinden sich bereits 2 Kunden im Geschäft bitte draußen warten, b Paris, Frühjahr 2020: >In Anbetracht der Umstände müssen wir leider das Restaurant schließen. Wir machen so bald wie möglich wieder auf. Das Team Mongoor

\footnotetext{
5 Die französischen Entsprechungen sind »ensemble, tous ensemble, solidaires«, >zusammen, alle zusammen, solidarisch $(\mathrm{pl})<$, Ausdrücke der Reziprozität »Protégeons-nous les uns les autres «, >schützen wir uns gegenseitig (die einen die anderen)<, oder auch der inklusive Imperativ 1.pl »Respectons encore les gestes barrières«, >respektieren wir weiterhin die Barrieregesten<. Die österreichischen Slogans lauten z.B. »Gemeinsam gegen das Coronavirus; Schau auf dich, schau auf mich.« In Schweden heißt es »... nu hjälps vi åt $\ll$ ( $>$ so ist uns allen geholfen $<$ ).
} 
hänge auf entsprechendem Plakatpapier konzipiert, oder wurden sie spontan mit der Hand auf unterschiedlichen Unterlagen ${ }^{6}$ geschrieben? Bei den handgeschriebenen Kommunikaten werden unterschiedliche Gestaltungsmittel eingesetzt wie Schnörkel, Verzierungen und verschiedene Unterstreichungen, manchmal auch Smileys. Die obigen Beispiele (Abb. 3) sind eher zurückhaltend und kommen ohne Verzierungen aus; die handschriftliche Form ist dabei von dem individuellen Schriftstil des schreibenden Inhabers oder Angestellten geprägt, wobei die Zeilen und Buchstaben etwas >tanzen< und die Interpunktion fehlt:

Corona-Kommunikate finden sich auf einer breiten Palette von Kommunikationsträgern: an und in Schaufenstern, an Häuserwänden und privaten Fenstern, auf improvisierten Trennwänden in Geschäften, Büros oder medizinischen Einrichtungen. Sie werden auf Aufsteller vor Geschäften gepinselt, auf den Gehsteig gesprüht oder geklebt, auch Leuchtplakate werden massiv eingesetzt.

Die ersten Corona-Kommunikate im März 2020 sind improvisiert, sie stellen eine Antwort auf die plötzliche, unerwartete und unerhörte Situation dar. Während der ganzen Dauer der Pandemie halten sich die frei formulierten Kommunikate, wenn die Verfasser*innen auf eine spezielle Situation reagieren. Doch schon bald nach Beginn der Pandemie kommen die ersten Vordrucke ${ }^{7}$ auf, die von öffentlichen und privaten Kommunikatoren verwendet werden können. Größere öffentliche und private Institutionen (Bahn, Geschäftsketten etc.) entwickeln ihre eigenen Kommunikationsformate. So werden in drei der beobachteten Ländern Piktogramme entworfen, die große Ähnlichkeit aufweisen, wie z.B. die Aufforderung, Abstand zu halten. Allerdings scheinen in Schweden Doppelpfeilpiktogramme auf dem Boden weniger üblich zu sein (s. Abb. 4).

a

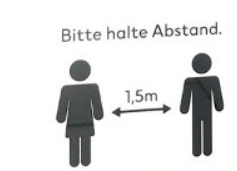

kenn möglich, kontaktlos.
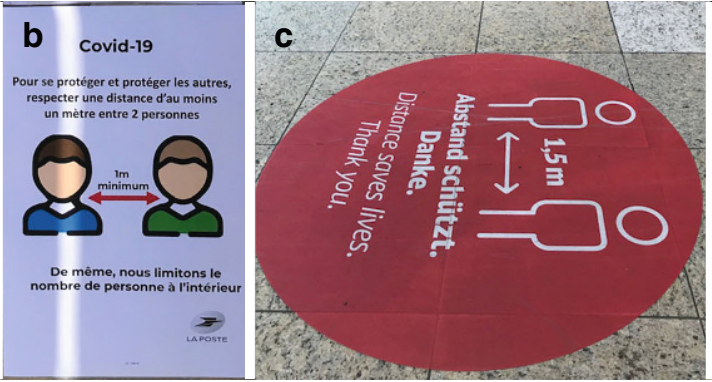

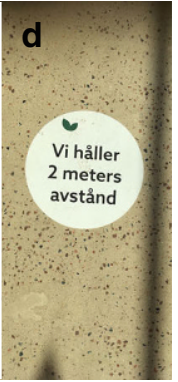

Abb. 4 Piktogramme des Abstandhaltens. a Österreich, b Frankreich, c Deutschland, Bodenmarkierung, d Schweden, Bodenmarkierung

\footnotetext{
${ }^{6}$ Z.B. auf Bettlaken, die aus dem Fenster gehängt werden: » 221 morts / en sortir sans sortir / merci aux soignants / rdv 20h« (Paris, April 2020) >221 Tote / Die Krise überstehen ohne rauszugehen / Dank an das Pflegepersonal / RV 20h «. Man erinnert sich, dass in Paris von Mitte März bis Mitte Mai die Bevölkerung jeden Abend gegen 20h an den offenen Fenstern stand und dem Pflegepersonal applaudierte.

${ }^{7}$ Hier einige Adressen für Selbstausdrucke:.https://www.santepubliquefrance.fr/maladies-et-traumatismes/ maladies-et-infections-respiratoires/infection-a-coronavirus/documents/depliant-flyer/covid-19-supportsd-information-grand-public.-catalogue-janvier-2021 (4.4.2021), https://www.rki.de/DE/Content/InfAZ/ N/Neuartiges_Coronavirus/Quarantaene/haeusl-Isolierung.html,. https://www.oesterreich.gv.at (4.4.2021).
} 


\subsection{Medialität}

Das entsprechende Klassifikationskriterium stützt sich auf die Medialität des Kommunikats. Aushänge kommen als rein schriftliche Kommunikate vor wie in Abb. 2, aber auch - wenn auch sehr viel seltener - als rein bildlich/figürliche. Rein bildlich über Piktogramme werden vor allem Abstandsregeln kommuniziert (Abb. 4 und 22). Mit einem Leuchtvideo wirbt der Künstlers Borat für das Tragen des Mund-NasenSchutzes (Nizza, Herbst 2020). Sehr viele Kommunikate sind jedoch multimodal, verbinden schriftliche und bildliche Elemente im Sinne von Sehflächen, die nach Ulrich Schmitz »gemeinsame Bedeutungseinheiten« bilden (Schmitz 2010, S. 3).

Ein Beispiel für die Klasse der kombinierten Schrift/Bild-Kommunikate ist der Aushang in Abb. 5a. Das Piktogramm (b) ist der große Blickfänger, der im Stil eines Gebots im Straßenverkehr in Deutschland gehalten ist. Es wird eingerahmt durch die verblose Ermahnung »Maskenpflicht« (a) sowie die entsprechende Bitte in Form eines Imperativs in der Höflichkeitsform (c). Schrift und Bild kodieren doppelt die neue Obligation, die (noch) keine Routine ist.

Das Kommunikat in Abb. 5b ist figürlich in Form einer Beethovenbüste mit Maske. Ursprünglich handelt es sich um ein Dekorationsobjekt, das durch die umgehängte Maske seinen semiotischen Status ändert und zu einem coronaspezifischen Kommunikat wird. Da die Büste im Schaufenster eines Musikalienhandels steht, ist hier zugleich eine gewisse Ironisierung der Hygienemaßnahmen zu erkennen.
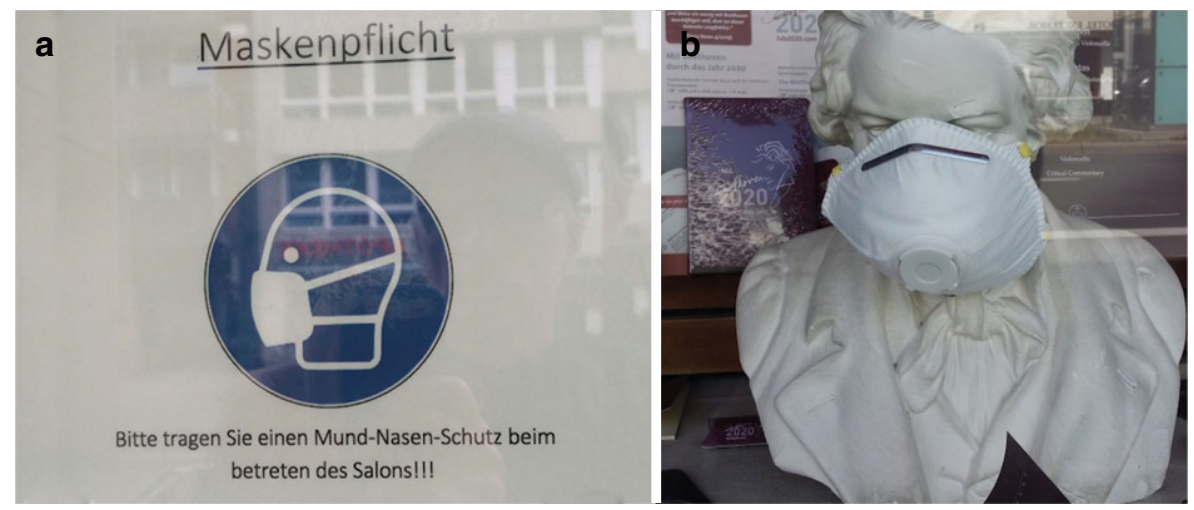

Abb. 5 Maske tragen. a Düsseldorf, Frühjahr 2020, Aushang an der Scheibe eines Friseursalons: (a) Maskenpflicht, (b) Piktogramm, (c) Bitte tragen Sie einen Mund-Nasen-Schutz beim Betreten des Salons, b Düsseldorf, 2020, Büste in einem Musikalienhandel 


\subsection{Lokalität}

Neben der grafischen oder sprachlichen Form der Kommunikate spielt der Ort ihrer Anbringung eine wichtige Rolle. ${ }^{8}$ Sie können, wie schon erwähnt, im Innenbereich, im Außenbereich oder im Übergang zwischen beiden, an Scheiben oder Eingängen positioniert sein (wie die liminalen Aushänge in Abb. 2 und 3). Das entsprechende Klassifikationskriterium ist dasjenige der Lokalität. Ein Aufsteller, der vor einem Fischgeschäft positioniert ist, zeigt entsprechend Spuren der Witterung, denen er ausgesetzt ist (s. Abb. 6). Die geäußerte Bitte ist auf einer Tafel vermerkt, die sonst wohl der Auflistung des Warenangebots dient. Der Gesichtspunkt der Lokalität wird im Abschnitt 8 weiter unter dem Aspekt der Arealität öffentlicher Kommunikate ausbuchstabiert.

Die Anbringung der Kommunikate variiert stark, je nachdem, ob sie vorwiegend expressiv, regulativ oder informativ sind (s. Abschnitt 5). Sie variieren in ihrer Permanenz, der Anbringungshöhe und in ihrem Ortsbezug.

Expressive Kommunikate sind weniger an einen bestimmten Ort oder Raum gebunden als regulative und informative. Sie erscheinen in verschiedenen Formaten und auf verschiedenen Trägern. Ein allgemeiner Dank an das Pflegepersonal oder die Mitarbeiter soll gut sichtbar sein und wird in einer bestimmten Höhe angebracht, während ein Dank an bestimmte Personen auch kleinformatig an eine Haustür geklebt werden kann.

Als Aufschrift auf einer der Zeltplanen bezeichnen informative Kommunikate Zelte als Test- oder Impfzentren, als Aushänge am Schaufenster einer Apotheke versichern sie die Leser*innen, dass er/sie sich in dieser Institution testen lassen kann. Regulative Kommunikate werden ebenfalls so angebracht, dass die Leser*innen spätestens beim Betreten eines Raumes wissen, dass sie die AHA-Regeln/gestes barrières befolgen müssen. Diese Kommunikate sind also so eng wie möglich an dem Bezugsobjekt bzw. Bezugsraum angebracht. Die Anbringungsweisen vor allem

\footnotetext{
Abb. 6 Schweden, 2020, Aufsteller vor einem Fischgeschäft: HALTEN SIE ABSTAND Kommen Sie nur zum Kaufen oder Essenbestellen herein. Kommen Sie nicht herein, wenn Sie sich nur umschauen möchten. Die Toilette ist nur für unsere Kunden, die anderen werden auf die öffentlichen Toiletten verwiesen (10-15 Meter nordwärts). HALTEN SIE ABSTAND
}

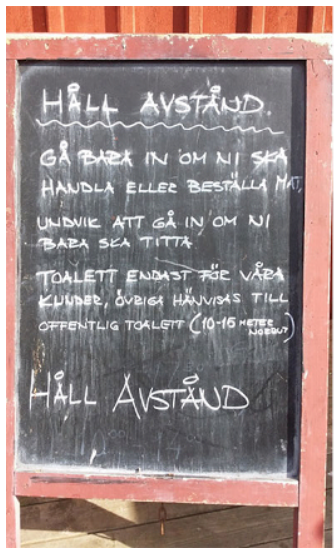

8 S. vor allem Bühler (1934/78 § 10,2), Androutsopoulos (2020), Auer (2010), Domke (2013), Scollon/ Scollon (2003). Zu deiktischen und temporal-aspektuellen Aspekten Behr (2005, 2019), zur Finitheit ortsgebundener Schriftlichkeit Hennig (2010). 
der regulativen Kommunikate sind improvisiert oder professionell, permanent oder zeitlich begrenzt - was zählt, ist, dass der Leser sie an den relevanten Orten zu relevanten Zeitpunkten lesen kann.

\section{Diskursive Funktionen der Kommunikate}

Die Corona-Kommunikate erfüllen verschiedene Funktionen, die in unserer Untersuchung im Vordergrund stehen. Die Kommunikate werden sowohl von Privatpersonen, von gemeinnützigen Organisationen und von Geschäftsinhabern als auch von verschiedenen Institutionen verfasst (öffentliche und private Transportunternehmen, Verwaltung, Bildungseinrichtungen etc.), daneben gibt es auch anonyme Verfasser.

\subsection{Expressive Funktion}

Expressive Elemente in Corona-Kommunikaten dienen dazu, den eigenen Emotionen angesichts der auferlegten Restriktionen oder der gesundheitlichen Situation Ausdruck zu geben. Die Palette umfasst sowohl positive als auch negative Einschätzungen und Emotionen, wie z.B. DANKBARKEIT gegenüber den Pflegekräften und systemrelevanten Arbeiter*innen:

(01) Merci à nos soignants et à tous ceux en 1ère ligne (handgeschriebenes Plakat, Paris, Juni 2020) ${ }^{9}>$ Dank an unsere Pflegekräfte und an alle der ersten Linie<

(02) S. Abb. 8: Wir sagen allen Helfern und unseren Teams DANKE!

DANKSAGUNGEN an treue Kunden an den Schaufenstern verschiedener Geschäfte oder auf städtischen Kommunikationstafeln, Frühjahr 2020:

(03) Merci à tous d'avoir illuminé les Champs-Elysées (Paris, November 2020). >Dank an alle, die die Champs-Elysées illumiert haben`

(04) Liebe Kunden. Wir möchten uns an dieser Stelle recht herzlich für Ihre Treue bedanken. Leider hat uns die Coronakrise fest im Griff und wir müssen daher die Trafik ab Montag den 27. April 2020 bis auf Weiteres geschlossen halten. [...] Ihr Trafik-Team (Wien, April 2020)

SOLIDARITÄT:

(05) Ensemble et solidaires. [Herz-Piktogramm] $1 €$ reversé à l'APHP sur chaque bouteille de rosé vendue jusqu'au 31/05/20. Déja $4564 €$ récoltés au 10/05/20 (Aufsteller vor einem Weinladen, Paris, Mai 2020) >Gemeinsam und solidarisch. $1 €$ überwiesen an die $\mathrm{APHP}^{10}$ für jede Flasche Rosé, die vor dem $31 / 05 / 20$ verkauft wird. Schon $4564 €$ gesammelt am 10/05/20

(06) S. Abb. 9 Gemeinsam gegen Corona

\footnotetext{
9 D.h. in erster Linie gegen den Feind Covid-19.

10 APHP = Assistance Hôpitaux Publics de Paris.
} 
Abb. 7 Paris, Frühsommer 2020, Aushang an einem privaten Fenster, >Kohle für das öffentliche Krankenhaus

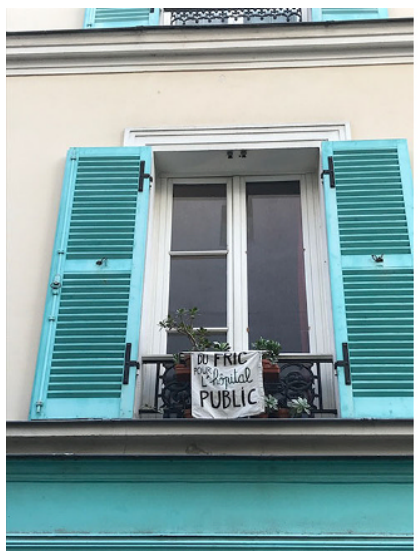

\section{WIEDERSEHENSFREUDE}

(07) Schön, Sie wiederzusehen! (Haan, Frühjahr 2020) ${ }^{11}$

(08) Nous sommes ravis de vous retrouver à partir du vendredi 05 juin à $12 \mathrm{~h}$. Vous nous avez manqué! We are so happy to meet you again from Friday 05th of june at noon. We missed you! (zweisprachiger Aushänger an einem Fenster des Café Les deux Magots, Paris, Frühjahr 2020) >Wir freuen uns Sie ab Freitag 05. Juni um $12 \mathrm{~h}$ wiederzusehen. Sie haben uns gefehlt!

EMOTIONALE REAKTIONEN, DIE IN FORDERUNGEN MÜNDEN:

(09) Du Fric pour l'hôpital public (Aushänger an einem privaten Fenster, Paris, Frühjahr 2020, Abb. 7) >Kohle für Krankenhäuser

(10) Unsere Eventbranche STIRBT! Regierungshilfe JETZT! (Straßenplakat der Vereinigung der Österreichischen Veranstaltungsdienstleister, Wien, Sommer 2020, Abb. 14b)

Zusammenfassend kann man sagen, dass die Unerhörtheit der Krise einen diskursiven Raum erfordert, in dem die im weitesten Sinne emotionalen Reaktionen ausgedrückt werden können.

\subsection{Regulative Funktion}

Regulative Corona-Kommunikate dienen dazu, einen Teil der Maßnahmen im öffentlichen Bereich konkret in Handlungen und Verhalten umzusetzen (s. Abb. 2), und zwar mit unmittelbarer oder zeitlich versetzter Wirkung. So sollen die Leser*innen konkret und punktuell zur Befolgung der Verordnungen aufgefordert werden, wie z.B. Abstand halten und Maske tragen; oder es werden ihnen Ersatzhandlungen angeboten, wie z.B. online-Kaufen statt im Geschäft.

11 Beleg von R. Behr, der hier gedankt sein soll.

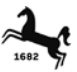


(11) Sehr geehrte Kundinnen, sehr geehrte Kunden, aufgrund gesetzlicher Bestimmungen dürfen diesen SB-Bereich nur 2 Personen gleichzeitig betreten. Bitte achten Sie zwingend auf einen Mindestabstand von 1,50 m. Verstöße können mit einem Bußgeld geahndet werden. (Bankfiliale, Düsseldorf, Mai 2020)

\subsection{Informative Funktion}

Informative Corona-Aushänge erscheinen in dem beobachteten Zeitraum etwas später, als es darum geht, den Vorrat an Gesichtsmasken anzugeben oder im öffentlichen Raum Test- oder Impfstellen zu kennzeichnen bzw. auf sie zu verweisen.

(12) Vente Masque chirurgical (Paris, Mai 2020, Aushänger an einem bureau de tabac) > Verkauf von chirurgischen Masken<

(13) Ici, dépistage Covid gratuit (Paris, März 2021, Zelt vor einer Apotheke) >Hier kostenfreie Covidtestung<

(14) Banner: [symbolisierte Spritze und Flasche mit Impfstoff]: Impfzentrum Düsseldorf (April 2021) Quelle: duesseldorf.de

\subsection{Mischformen}

Man kann also festhalten, dass die von uns beobachteten Kommunikate drei Zwecke haben: regulativ das unmittelbare oder zeitlich versetzte Handeln und Verhalten dahingehend zu beeinflussen, dass das routinehafte Handeln und Verhalten zugunsten einer der Krise angepassten Weise geändert wird; informativ auf Vorrichtungen hinzuweisen, die das krisengerechte Verhalten erlauben; expressiv die durch die Krise ausgelösten Emotionen zur Sprache zu bringen. Der regulative Aspekt ist dominant, kann aber indirekt sein, wenn die Ausdrucksformen gemischt sind.

Mischformen von expressiven Elementen mit regulativen bestehen in Botschaften wie »Danke, dass Sie Maske tragen/Abstand halten «. Hier mischen sich Emotion, also Dankbarkeit, und Regulation, d.h. Einforderung des Maskentragens bzw. Distanzhaltens. Ebenso häufig sind Mischformen von informativen und regulativen Elementen:

(15) Votre gare vous facilite l'hygiène. Un distributeur de gel hydro-alcoolique est à votre disposition dans cette gare. SNCF (Paris, Juni 2020, Informationsplakat im Bahnhof Montparnasse)

$>$ Hygiene im Bahnhof leicht gemacht. Ein Spender mit hydro-alkoholischem Gel steht Ihnen in diesem Bahnhof zur Verfügung<. (Abb. 19a)

(16) Wichtige Information!!! Zutritt nur mit Einkaufshelfern! Vielen Dank für Ihr Verständnis! [Symbol eines Einkaufskorbs mit Unterschrift: Nur mit Einkaufshelfer!] (Woolworth-Filiale Düsseldorf, Mai 2020) 


\section{Kommunikate als Texte}

\subsection{Frame als Grundlage der Botschaft}

Am Beispiel der Kommunikate, die zum Maskentragen auffordern, wollen wir einige textuelle Eigenschaften der Kommunikate beschreiben. ${ }^{12}$

Wir modellieren das dahinterstehende Frame auf allgemeiner Ebene folgendermaßen:

OBLIGATION [Handlung (AGENS, Zeit, Ort) $]_{\text {Anbringungsort/Geltungsort }}$

Dieses Schema lässt sich wie in Tabelle 1 auffüllen. Die Obligation, einen MundNasen-Schutz zu tragen kann dann explizit gemacht werden:

(17) In (dem Viertel X) der Stadt Y müssen Personen ab 11 Jahren/Sie von $6 \mathrm{~h}$ bis $19 \mathrm{~h}$ auf der Straße und in kommerziellen oder institutionellen Räumen einen MNS tragen.

Sie kann aber auch nur partiell versprachlicht werden (vgl. Abb. 2; Abb. 4). In der Tat explizieren situierte (Scollon/Scollon 2003) Kommunikate zumeist nur Teile des Frames, der Rest wird über Lektüresituation und Lektürekontext (s. o. Abschnitt 3.2) geübt mitverstanden. Ein Kommunikat wie »[Piktogramm] Port du masque obligatoire «/>Tragen eines MNS obligatorisch< realisiert explizit die Komponenten

[OBLIG, MNS tragen/porter un masque],

die Komponenten

[AGENS, Zeit, Ort]

hingegen werden von den Leser*innen im Augenblick des Lesens ergänzt, ebenso wie die indexikalische Beziehung zwischen Anbringungs- und Geltungsort.

Die Maskenpflichtkommunikate können über die minimalen Elemente hinaus andere Elemente enthalten, sie können diverse Anreicherungen und Perspektivierungen erfahren: einen (deiktischen) Hinweis auf den Geltungsraum (»ici, le port du masque est obligatoire« > hier herrscht Maskenpflicht $<$ ), die Finalität (»pour la sécurité de

Tab. 1 Frame für Aufforderungen

\begin{tabular}{|c|c|c|c|}
\hline \multirow[t]{2}{*}{ OBLIG Handlung } & AGENS Zeit & Ort & $\begin{array}{l}\text { Anbringungs-/ } \\
\text { Geltungsort }\end{array}$ \\
\hline & Indexikalisch & & Indexikalisch \\
\hline $\begin{array}{l}\text { OBLIG einen Mund- } \\
\text { Nasen- } \\
\text { Schutz tra- } \\
\text { gen }\end{array}$ & $\begin{array}{cl}\text { Leser*in } & \text { in den von den Be- } \\
& \text { hörden vorgeschrie- } \\
& \text { benen Zeiträumen }\end{array}$ & $\begin{array}{l}\text { an den von den } \\
\text { Behörden vorge- } \\
\text { schriebenen Orten }\end{array}$ & $\begin{array}{l}\text { Anbringungs- } \\
\text { ort verweist auf } \\
\text { Geltungsort }\end{array}$ \\
\hline
\end{tabular}

12 Offizielle, genormte Aufforderungen zum Maskentragen, z.B., können vom zuständigen Ministerium oder einer Wirtschaftskammer verfasst sein, die von Behörden und Institutionen ebenso verwendet werden wie von Geschäftsinhabern. Diese Praxis lässt sich in Frankreich, Deutschland und Österreich beobachten, sobald a) die Vorschrift/Empfehlung erlassen ist und b) solche Formate angeboten werden. In dem beobachteten Zeitraum besteht jedoch in Schweden weitgehend keine Maskenpflicht. Die MaskenpflichtBotschaften erscheinen im Straßenbild auf Trägern der städtischen Kommunikation sowie in den Vitrinen und Fassaden der Geschäfte, Restaurants und Institutionen. Die Zielrichtung ist insgesamt regulativ. Sie konkurrieren oder kombinieren sich mit frei gestalteten Aufforderungen zum Maskentragen. 
tous « > für Ihre und unsere Gesundheit $<$ ) bzw. die Integration in eine von allen getragene Strategie (»Ensemble, faisons bloc contre le coronavirus « >Gemeinsam gegen den Coronavirus $<$ ). Sie können auch Höflichkeits- oder Indirektheitsmarker enthalten ( $>$ bitte $</>$ please $<$; 1. Person sg oder pl). Die Abfolge und Kombination der Elemente, die quasi als Textbausteine fungieren, sowie ihre Kombination mit Piktogrammen erfolgt relativ frei.

\subsection{Sprechaktkategorien und sprachliche Formen}

Die Kommunikate setzen sich aus Sprechakten unterschiedlicher Kategorien zusammen, wobei bestimmte Texte auch mehrere Sprechakte enthalten können.

Abb. 6 z.B. zeigt den schon erwähnten Aufsteller vor einem Fischgeschäft mit Imbiss, dessen Botschaft dominant direktiv ist: >Halten Sie Abstand $<$. Der Text beginnt mit zwei direktiven Sprechakten ( $\mathrm{a}>$ Kommen Sie nur ...< und $\mathrm{b}>$ Kommen Sie nicht ...<), welche den Zugang zum Geschäft regeln. Die deklarativen Sprechakte (c >die Toilette ist nur... $<$ und $\mathrm{d}>$ die anderen ...<) dienen ebenfalls dazu, die Zahl der Personen in dem Geschäft zu reduzieren. Indem sie Aussagen über ein bestimmtes Element (hier die Toilette) machen, verbieten sie den Personen, die nicht Kund*innen sind, die Benutzung eben dieses Elements und verringern damit den Publikumsverkehr. Man kann (c, d) auf einer weiteren Analyseebene als eine Variation der direktiven Sprechakte auffassen.

In Abb. 8 liegt die erwähnte Mischform von regulativer und expressiver Funktion vor, die hier auf die entsprechenden Sprechakttypen bezogen werden kann: Es erfolgt zunächst ein expressiver Sprechakt des Dankens, wobei der Gegenstand des Danks nicht genannt, möglicherweise aber in der Nennung der Adressat*innen impliziert ist: Den Helfern und dem Team wird für das Helfen gedankt. Der folgende Direktiv, ein Appellieren, enthält lediglich die Prädikation, nämlich die Krise zu bewältigen, wobei die Handelnden ebenfalls in dem Adverb >gemeinsam< impliziert sind.

Persuasive Sprechakte sind von einer gewissen Emotionalität geprägt. Auf einem Aushang an einer U-Bahn-Haltestelle, der mehrere Anweisungen und Bitten bezüglich der Corona-Etikette enthält, findet sich am Schluss eine Art Goldene Regel:

\footnotetext{
Abb. 8 Düsseldorf, Frühjahr 2020, Aufsteller an einer Tankstelle: Neues von Shell WIR SAGEN ALLEN HELFERN UND UNSEREN TEAMS DANKE! GEMEINSAM DIE KRISE BEWÄLTIGEN GO WELL [Piktogramm Shell]
}

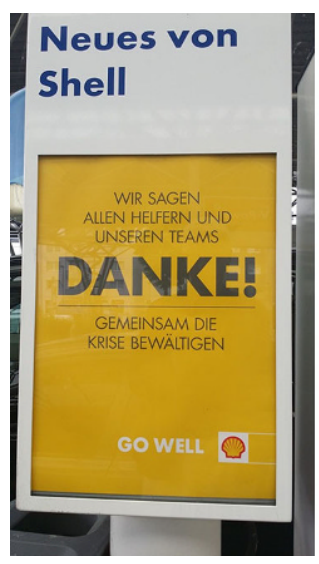


$>$ Wenn alle helfen, ist allen geholfen $<$. Das individuelle Helfen wird den positiven kollektiven Folgen gegenübergestellt und mit diesen in einer wenn-dann-Beziehung verbunden. Eine regulativ-expressive Mischform liegt auch hier vor.

Die sprachliche Form des Kommunikate spiegelt die Entscheidungen wider, die die Autoren bei der Versprachlichung getroffen haben: Persönliche oder unpersönliche Formulierungen, Höflichkeitspartikeln, Verweis auf räumliche und zeitliche Geltungsbereiche sowie auf die Maßnahmen der Regierung. Da sie auf unterschiedlichen Ebenen spielen, sind sie untereinander kombinierbar. Dazu kommen die oben schon erwähnten Anreicherungen der Grundschemata.

Persönliche Formulierungen enthalten Personalpronomen, Possessiva der 1. oder 2. Person, und in der Regel konjugierte Verben:

(18) Wir reinigen, damit du gesund bleibst. (Abb. 19b)

(19) Votre gare vous facilite l'hygiène. >Im Bahnhof Hygiene leicht gemacht.< (Abb. 19a)

(20) Bitte tragen Sie einen Mund-Nasen-Schutz beim Betreten des Salons (Abb. 5a)

(21) Restez chez vous! >Bleiben Sie/bleibt zu Hause!< (Abb. 15b)

Unpersönliche Formulierungen enthalten modale Infinitive und/oder nominale Ausdrücke, oder sie sind in der 3. Person gehalten; sie sind modalisierbar (25) und eignen sich u.a. dazu, in komprimierter (von Polenz 1988) Form lediglich die Art und Weise einer geforderten Handlung anzugeben (26):

(22) Maskenpflicht (Abb. 5a)

(23) Port du masque obligatoire >Maskenpflicht< (Abb. 13)

(24) Merci à nos soignants >Dank an unsere Pfleger< (Abb. 10)

(25) Bitte nach Möglichkeit einzeln eintreten (Abb. 3a)

(26) Bitte nur mit Abstand und Gesichtsmaske! (Abb. 2)

Die Aufforderungen können begründet werden (»pour la sécurité de tous/pour notre santé à tous« >für unser aller Sicherheit/Gesundheit<, »zum Schutz unserer Kunden und Mitarbeiter«, »zu Ihrer und unserer Sicherheit«), oder es wird für die Einhaltung der Vorschrift gedankt (»merci de votre compréhension« >Danke für Ihr Verständnisく, »vielen Dank und bleiben Sie gesund«).

\subsection{Slogans}

Schließlich wird auch mit kurzen Slogans gearbeitet, die entweder isoliert in einer Schrift/Bild-Kombination vorkommen oder als Zusatz auf anderen Aushängen platziert werden. Der folgende Slogan (Abb. 9), der in unterschiedlichen Kontexten immer wiederkehrt, kann dabei als Versuch gelten, so etwas wie eine Routine in das krisenhafte Geschehen hineinzubringen.

Grafisch auffallend ist hier das stilisierte Herz, das zugleich einen Pfeil nach unten darstellt, als Symbol für sinkende Infektionszahlen. Die Wahl einer weißen Schrift auf dunklem Hintergrund steigert die Dramatik der Bildgebung. Dies wird unterstützt durch die verblose Form des Slogans, die der Botschaft einen komprimierten Charakter verleiht. 
Abb. 9 Slogan in unterschiedlichen Kontexten

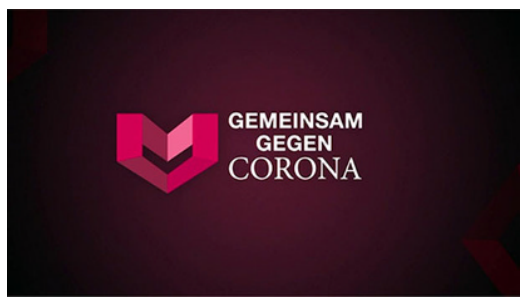

Auf französischen Aushängen findet sich die Devise »protégez-vous et protégez les autres « (>schützen Sie sich und schützen Sie die anderen $<$ ) häufig auf der obersten oder der untersten Zeile, allerdings ohne visuelle Unterstützung.

\section{Historizität der Corona-Kommunikate}

\subsection{Phasen der Krise}

Die Corona-Krise durchläuft verschiedene Phasen. Von Mitte März 2020 bis März 2021 lassen sich en gros drei Phasen ausmachen. Die erste Phase entspricht dem Ausbruch der Pandemie und den ersten restriktiven Maßnahmen, also dem Frühjahr 2020. Die zweite Phase entspricht den allgemeinen Lockerungen nach der restriktiven Phase, also dem Sommer 2020. Die dritte Phase hingegen entspricht einer chronischen Phase, also der Zeit ab dem Herbst 2020: Die gesundheitliche Lage verschlechtert sich wieder, ähnliche Einschränkungen wie schon im Frühjahr und vorsichtige Lockerungen lösen einander ab. Es entsteht eine Art Gewöhnungs- oder Déjà-vu-Effekt. ${ }^{13}$

\subsection{Realisierung der diskursiven Funktionen in den Phasen}

Die erste Phase ist die Phase der akuten Not, der Überraschung, der $>$ Schockstarre< (>sidération<). Verständlicherweise sind die emotionalen Aspekte sehr wichtig. Die regulativen Aspekte sind noch nicht so ausgefeilt und stabil wie im Herbst und Winter 2020. In der ersten Phase dominieren drei Aspekte, die sich in den Kommunikaten niederschlagen:

- Ausdruck von Emotionen, Appellen, Forderungen ${ }^{14}$

- Information über die Pandemie, Schöpfung sowie die konkrete, jeweils punktuelle Umsetzung neuer sozialer Normen, die zur Vermeidung von Ansteckungen dienen sollen $^{15}$

\footnotetext{
13 S. hierzu die Beobachtungen zur Chronifizierung der Krise von Dreesen/Pohl (2020).

14 In Frankreich: Forderung nach Masken und Tests, Unterstützung für das Gesundheitswesen, Anerkennung, Dankbarkeit und Respekt für diejenigen, die während des Lockdowns die Versorgung der Bevölkerung gewährleisteten, Solidarität mit sozial Schwachen und durch den Lockdown Gefährdeten.

15 Aufrufe, zu Hause zu bleiben; Restriktionen des Publikumsverkehrs in Geschäften und Institutionen, im Transportwesen; AHA-Formel bzw. >gestes barrières< (>Barriere-Gesten $<$ ).
} 
- Aufrechterhaltung der kommerziellen sowie der im weiten Sinne sozialen Beziehungen unter den Bedingungen der Pandemie ${ }^{16}$

Sie zeigen sich in den folgenden Beispielen:

(27) Pour respecter les directives du gouvernement, notre boutique est fermée. Vous pouvez commander nos produits sur www.xxxxx.fr. Les frais de port sont offerts! Profitez-en! (Maschinengeschriebener Aushang, Geschäft, Paris, April 2020)

$>$ Um die Anweisungen der Regierung zu respektieren, ist unsere Boutique geschlossen. Sie können unsere Produkte auf www.xxxxx.fr bestellen. Die Portokosten übernehmen wir! Nutzen Sie die Gelegenheit!<

(28) Merci! Maintenir notre activité, malgrés [sic] une période très compliquée est un défi. Chaque jour, vous nous remerciez, à notre tour de vous dire Merci. Aujourd'hui vos courses chez vos artisans sauveront l'activité de demain. L'équipe du Grenier à pain (Handgeschriebener Aushang, Bäckerei, Paris, April 2020) >Danke! Unsere Aktivität aufrechtzuerhalten, trotz der sehr komplizierten Zeiten, ist eine Herausforderung. Jeden Tag danken Sie uns, jetzt ist es an uns, Ihnen Danke zu sagen. Ihre heutigen Einkäufe bei Ihren Einzelhändlern sichern die Aktivität von morgen.<

(29) Bitte helfen Sie mit, sich, Ihre Mitmenschen und unsere Mitarbeiter mit einfachen Regeln zu schützen. (Woolworth-Filiale, Düsseldorf, Mai 2020)

Die zweite Phase ist die des Aufatmens: Der Lockdown ist überstanden. Wirtschaftlich sind alle betroffen gewesen, nun können nach und nach die Aktivitäten wiederaufgenommen werden, wenn auch unter bestimmten Auflagen. Eine Vielzahl expressiver Kommunikate zeugt von der Freude, Kunden und Mitmenschen wiederzusehen, wieder nicht-digitale zwischenmenschliche Kontakte zu pflegen - kurz, (fast) wieder $>$ normal $<$ zu leben.

Mittels expressiver Kommunikate wird der Freude des Wiedersehens Ausdruck gegeben:

(30) Wir freuen uns, dich wiederzusehen (Geschäftsauslage, Wien, Sommer 2020)

(31) Liebe Gäste! Es ist soweit. Wir sind ab morgen (Montag 11.05.20) wieder da $\rightarrow$ aber mit 1,5 m Abstand. Wir freuen uns auf Euch! Ihr Lo-Zibellino Team (Restaurant, Düsseldorf, Frühsommer 2020)

(32) Re-bonjour les blagues de papa au café (Bistrot-Tisch, Paris, Frühsommer 2020) $)^{17}$

$>$ Da sind sie wieder, Papas Kaffeehauswitze<

\footnotetext{
16 Umsteigen auf digitales Einkaufen, Solidarität mit dem Restaurations- und Kultursektor.

17 Der Bistrot-Tisch zeigt unter einer Glasplatte eine ganzflächige Werbeanzeige für eine Kreditkarte. Neben dem Hauptslogan, der einen lachenden älteren Herren zeigt, findet sich in kleinerer Schrift die Werbung für die Kreditkarte »Bonjour le paiement sans contact« >Willkommen dem kontaktlosen Bezahlen $50 €$ heraufgesetzt wurde. Die Werbeanzeige nimmt somit auf zwei aktuelle Ereignisse Bezug, die eng mit der Corona-Krise verbunden sind, nämlich auf die Heraufsetzung der Zahlungspauschale während des französischen Lockdowns und auf die Wiederöffnung der Cafés im Mai 2020.
} 
Abb. 10 SNCF, Frühsommer bis Herbst 2020, Bahnhöfe in Frankreich, >DANK all jenen unsichtbaren Händen, jenen Beziehungen, die nicht unterbrochen sind, all jenen, die unser Gleichgewicht aufrecht erhalten und uns erlaubt haben, nicht den Faden unseres Lebens zu verlieren.<

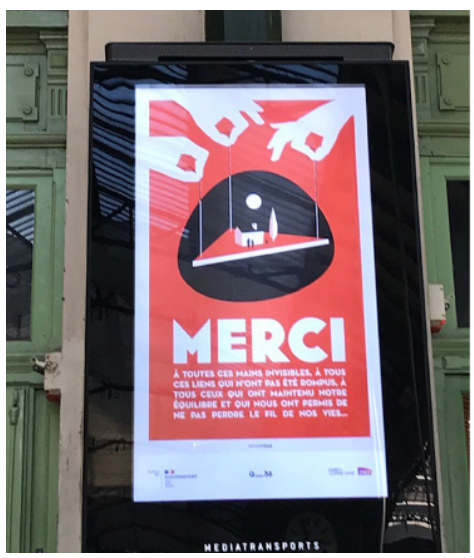

(33) Bienvenue dehors, vous nous avez manqué (Straßenkommunikation, Paris, Frühsommer 2020) >Willkommen draußen, Ihr habt uns gefehlt<

Privatpersonen und Institutionen machen ihre Danksagungen im öffentlichen Raum sichtbar:

(34) Merci à nos soignants et à tous ceux en 1ère ligne (privates Plakat an einer Gartenmauer, Paris, Frühsommer 2020) (s. auch Abb. 10)

>Dank an unser Pflegepersonal und an alle der ersten Linie [des Kampfes gegen das Virus]<

Gesundheitliche Auflagen sind weiterhin gültig und werden über Corona-Kommunikate eingefordert: Abstand halten, Desinfektionsgel benutzen, digitale Zahlungsmittel bevorzugen, gegebenenfalls Maske tragen. Die Aushänge in den Vitrinen der Geschäfte und Restaurants (s. Abb. 11) werden um zusätzliche Informationen bereichert, welche die neuen Regeln des Publikumsverkehrs in Erinnerung rufen; Administrationen und Institutionen (z.B. Gewerbekammern) sind jetzt in der Lage, Vordrucke anzubieten oder durchzusetzen.

Im Sommer 2020 ändert sich die Lage allmählich, denn mehrere Probleme treten nun gleichzeitig auf. Die Wegwerfmasken werden zu einem Müllproblem (Abb. 12). Die neuen Botschaften sind in die habituellen Kommunikationsformate integriert beides lässt auf ihre Permanenz schließen.

Ab dem Herbst 2020 steigen die Infektionszahlen erneut stark an, was sowohl Reisebeschränkungen, als auch neue Restriktionen, Ausgangssperren, Lockdowns nach sich zieht. Die Schließung von Schulen, Universitäten, kulturellen Einrichtungen, Dienstleistungsbetrieben und einer Reihe von anderen Geschäften, sowie die Aufforderung zu Home-Office wird von den Ländern jeweils spezifisch gehandhabt. Alles wird öffentlich diskutiert, und schlägt sich deshalb kaum in anders gestalteten oder neuen Corona-Kommunikaten nieder, die weiterhin der punktuellen Information und Regulation dienen. Die Wiedereinführung von Restriktionen wird als Wiederholung erlebt, der die Betriebe und Institutionen mit den im Frühjahr 

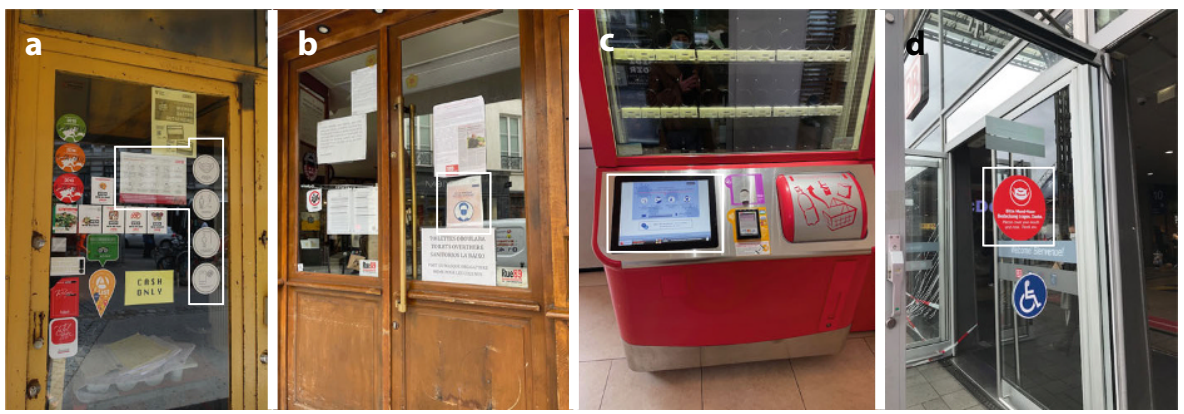

Abb. 11 Corona-Kommunikate neben anderen, installierten Kommunikaten. a Wien, 2020, Restaurant, b Paris, 2020, Restaurant, c Bahnhof Nizza, 2020, Automat, d Bahnhof Köln, 2020, Aufzug
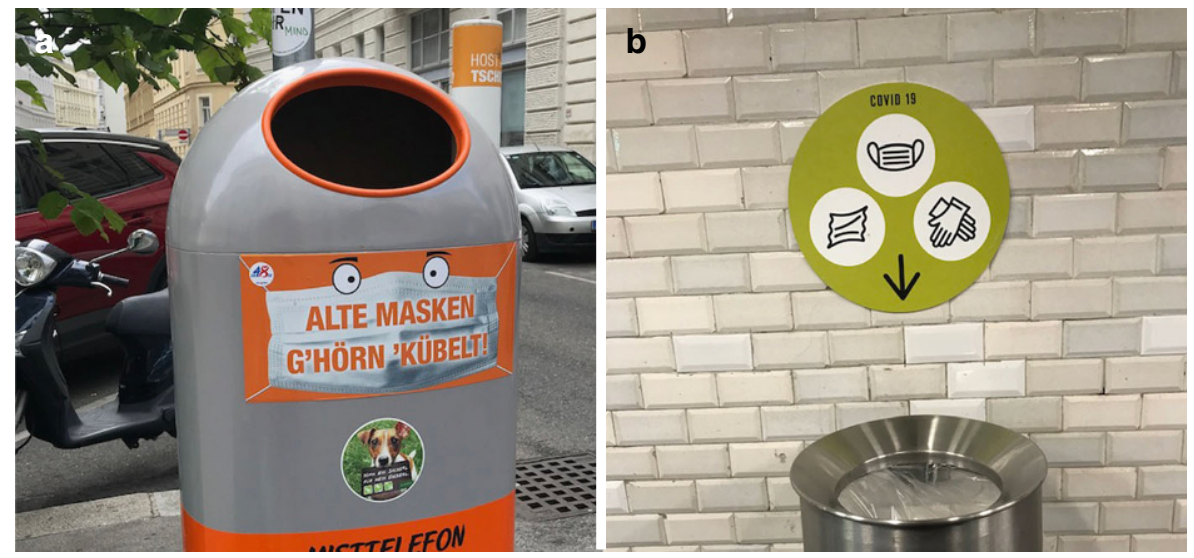

Abb. 12 Aufforderungen, die Masken richtig zu entsorgen. a Wien, Sommer 2020: ALte MASKEN G'HÖRN 'KÜBELT! b Paris, ab Frühling 2020, Métro: COVID 19 [Piktogramm Maske] [Piktogramme Papiertaschentuch, Handschuh]

erworbenen Routinen begegnen können. Diese werden aktualisiert, vielleicht auch mit Kommentaren angereichert oder in Perspektive gesetzt:

(35) Une nouvelle fois, vos boutiques EMAROBE \& EMAROBE BAZAR doivent se résoudre à fermer. (Bekleidungsgeschäft, Paris, während des NovemberLockdowns 2020)

>Erneut müssen sich Ihre Boutiquen EMAROBE \& EMAROBE BAZAR dazu durchringen, zu schließen. $<18$

$>$ Click\&collect< erlebt einen neuen Aufschwung, mit entsprechenden Aushängen. Die Corona-Kommunikate zu den Hygieneregeln und den Vorgaben des Publikums-

18 Hier der ganze Text des Aushangs: »Une nouvelle fois, EMAROBE \& EMAROBE BAZAR doivent se résoudre à fermer. Néanmoins, nous allons continuer à vous faire découvrir notre sélection sur Instagram \& Facebook, par mail xxx@xxx, ou appelez-nous au xxxx. Nous mettons également en place des rendezvous Facetime. Les retraits de vos commandes pourront se faire en boutiques, points relais partenaires ou en livraison à domicile. PRENEZ SOIN DE VOUS!«. >Erneut müssen sich Ihre Boutiquen EMAROBE 
Abb. 13 Nizza, Oktober 2020, Eingang einer Kirche. a $>$ Maskenpflicht $<$ [Piktogramm]

b> Verstärkte Sicherheit I Attentatsrisiko<. Großes Schild links [Piktogramm] An der Tür, rechts [Piktogramm Dreieck]

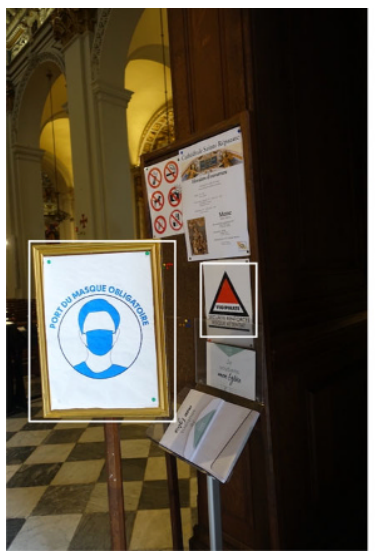

verkehrs werden zu neuen Quasi-Routinen - so präzisieren die Kommunikate ständig die jeweils geltenden Öffnungszeiten und Zulassungszahlen. Inhaltlich sind sie flexibel und werden den wechselnden Vorgaben angepasst, formal haben sie ihre Formen gefunden.

Daneben stellt man fest, dass sich alte und neue Risiken überlagern. So finden sich nach dem Nizzaer Attentat vom Oktober 2020 Corona- und Vigipirate-Kommunikate nebeneinander (s. Abb. 13). Fordern Corona-Kommunikate zu bestimmten Handlungen bzw. Verhaltensformen auf, um sich und andere vor dem unsichtbaren Virus zu schützen, so fordern Vigipirate-Kommunikate die Rezipienten auf, wachsam zu sein und materielle Anzeichen für Gefahren zu erkennen.

Insgesamt scheint sich die Einhaltung der über die Kommunikate vermittelten Vorschriften auf der Ebene des Verhaltens im Straßenverkehr einzuspielen - die Mehrheit hält sich eher mehr als weniger an die Regeln. Expressive Elemente sind dann zwar noch möglich und sozial wohl auch erwünscht als Abfederung der restriktiven Vorschriften, sie haben aber nunmehr den Stellenwert rhetorischer Elemente und haben als solche eine nicht zu unterschätzende soziale Nützlichkeit.

\section{3 Überblick über die Korrelationen zwischen Phasen und Ausprägungen der Diskursfunktionen}

Betrachtet man die Corona-Kommunikate zwischen März 2020 und März 2021, so stellt man fest:

- die expressiven Kommunikate werden nur in den beiden ersten Phasen >erfunden<; die meisten verschwinden wieder, einige wenige bleiben länger im öffentlichen Raum sichtbar,

\& EMAROBE BAZAR dazu durchringen, zu schließen. Trotz alledem werden wir Ihnen weiterhin unsere Kollektion auf Instragram \& Facebook vorstellen, per mail xxx@xxx, oder rufen Sie uns an unter xxxx. Wir werden ebenfalls Treffen per Facetime organisieren. Sie können Ihre Bestellungen in der Boutique oder an Partner-Relaispunkten abholen, oder aber wir liefern direkt. Passen Sie gut auf sich auf!<. 
- die regulativen Kommunikate werden ab April immer professioneller; neben handschriftlichen oder selbst ausgedruckten Botschaften setzen sich die offiziellen Aushänge und Formulierungen immer mehr durch,

- informative Kommunikate sind in allen drei Phasen präsent, sie werden vermehrt eingesetzt, um Test- und Impfstellen zu identifizieren,

- die Kombination Expressivitä//Information plus Regulation etabliert sich als eines der kommunikativen Muster.

\section{Arealität öffentlicher Kommunikate: Geltungsraum und Reichweite}

Zu Beginn des Beitrags hatten wir auf die Eigenschaft der Arealität der untersuchten Kommunikate hingewiesen. Es soll nun geklärt werden, was man sich genauer darunter vorstellen kann. Mit Peter Auer (2010) hatten wir festgestellt, dass der öffentliche Raum ein durch spezifische Handlungsroutinen gekennzeichneter Ort ist. Das Durchbrechen oder das krisenhafte Fehlen einer solchen Routinestruktur führt an spezifischen Orten zu öffentlicher Verwendung von Schrift - die hier unter dem Aspekt der Corona-Kommunikate untersucht wurde. Über die bestehenden Beschreibungsansätze hinaus, beispielsweise im Rahmen der linguistic-landscapeForschung, sind wir der Meinung, dass die Eigenschaft der Arealität für ein vollständiges Erfassen der Lektüresituation und ihrer Bedingungen unverzichtbar ist. Unter Arealität werden die im weitesten Sinne räumlichen Eigenschaften der Kommunikate verstanden. Sie betreffen den spezifischen Ort ihrer Anbringung oder Aufstellung, ihre räumliche Beziehung zu den jeweiligen Rezipient*innen, den >Befolgungsraum< von Anweisungen sowie im weiteren Sinne die Reichweite der aufgestellten Regelungen.

Dass Raum und Räumlichkeit eine essenzielle Bedingung für jegliches Erkennen und Handeln darstellen, ist Disziplinen- und Schulen-übergreifend unstrittig. So ist für Immanuel Kant die Vorstellung des Raumes (neben derjenigen der Zeit) eine Möglichkeitsbedingung von Erkenntnis. Sie wird folgendermaßen charakterisiert: »Der Raum ist eine notwendige Vorstellung, a priori, die allen äußeren Anschauungen zum Grunde liegt. [...] Er wird also als die Bedingung der Möglichkeit der Erscheinungen, und nicht als eine von ihnen abhängende Bestimmung angesehen [...] (KrV, B 38/39; A 24; Kant 1974) Anknüpfend an die Erkenntnistheorie der Phänomenologie Edmund Husserls entwickelte sich eine Phänomenologie der Lebenswelt von handelnden Individuen, die auf der Basis einer - sozusagen apriorisch selbstverständlichen Wirklichkeit agieren. Im Sinne von Alfred Schütz und Thomas Luckmann kann dabei von folgender Voraussetzung ausgegangen werden: »Nur in der alltäglichen Lebenswelt kann sich eine gemeinsame kommunikative Umwelt konstituieren. « (Schütz/Luckmann 1979, S. 25) Ein wesentlicher Bestandteil dieser kommunikativen Umwelt ist für Schütz/Luckmann die »räumliche Aufschichtung der alltäglichen Lebenswelt « (ebd., S. 63). Der uns als in der Lebenswelt agierenden Personen umgebende Raum ist strukturiert in verschiedene Ebenen oder Schichten, je nachdem, ob er für uns näher oder ferner ist. George Herbert Mead, auf den sich Schütz/Luckmann in ihrer Argumentation beziehen, hatte unterschieden zwischen verschiedenen Zonen des menschlichen Handelns, und zwar einerseits eine manipu- 
lative Zone, die unmittelbar wahrgenommen und manipuliert werden kann, und eine Zone der Ferndinge, die zwar gesehen, aber nicht mehr manipuliert werden können. (vgl. Mead 1938, S. 124f.) Im Gegensatz zu Mead trennen Schütz/Luckmann die uns umgebende Lebenswelt in drei Schichten auf, die sie mit dem Begriff der Reichweite beschreiben: Es geht um eine Welt in aktueller Reichweite, einen »Sektor der Welt, der meiner unmittelbaren Erfahrung zugänglich ist « (Schütz/Luckmann 1979, S. 64); andererseits um eine Welt in potentieller Reichweite, die sich in eine Welt wiederherstellbarer Reichweite gliedert - einen »Sektor, der eben noch in meiner aktuellen Reichweite war « (ebd., S. 65) und in eine Welt erlangbarer Reichweite eine »Welt, die nie in meiner Reichweite war, die aber in sie gebracht werden kann« (ebd., S. 66).

Die Schütz/Luckmannsche Unterscheidung von lebensweltlichen Wirklichkeitsformen, die eine aktuelle und zwei, jeweils in die Vergangenheit und in die Zukunft weisende potenzielle Reichweiten annimmt, ist in diesem phänomenologisch geprägten Ansatz auf das wahrnehmende und handelnde Individuum bezogen. Es geht um die Reichweite meines Erlebens und Agierens.

Der in diesem Beitrag vertretene Begriff der Arealität dreht gleichsam die Perspektive um und nimmt die Modi der Reichweiten von Kommunikaten selbst, also in phänomenologischer Sicht der wahrzunehmenden, kommunikativen Zeichengebilde, in den Blick. Wie greifen sie in die räumliche Aufschichtung der alltäglichen Lebenswelt ihrer Rezipient*innen ein? Bei der Beantwortung dieser Frage nehmen wir in Analogie zu Schütz/Luckmann ebenfalls unterschiedliche Reichweiten an, die sich jedoch auf die Beziehung des jeweiligen Kommunikats zu den intendierten Rezipient*innen richten. Wir unterscheiden drei Typen von Reichweiten, wobei diese als Geltungsbereiche gefasst werden können: eine allgemeine, nicht näher bestimmte und eine begrenzte Reichweite, wobei letztere wiederum unterteilt wird ein eine relativ weite und eine engbegrenzte Reichweite. Jeder dieser Typen wird wiederum in drei Kategorien unterteilt, somit ergeben sich insgesamt neun Kategorien (s. Tabelle 2), die im Folgenden erläutert werden.

Diese Reichweiten weisen durch ihre arealen Eigenschaften nicht nur Spezifika in Termini der Entfernung auf, sondern auch in der spezifischen Art der Lektüresituation durch die Rezipient*innen. Vor allem die Kommunikate mit eng bestimmter Reichweite erfordern bisweilen synchron zur Lektüresituation eine bestimmte Positionierung oder eine Verhaltensweise, während die Kommunikate mit weiter oder allgemeiner Reichweite keine Angaben zur Position oder zum Verhalten in der Lektüresituation machen. So verlangen Aufkleber auf dem Boden mit der Aufschrift

Tab. 2 Areale Reichweite der Corona-Kommunikate

\begin{tabular}{lll}
\hline $\begin{array}{l}\text { Areale Reichweite } \\
\text { allgemein }\end{array}$ & $\begin{array}{l}\text { begrenzt } \\
\text { weit begrenzt } \\
\text { Kategorien 4-6 } \\
\text { Kategorien 1-3 }\end{array}$ & $\begin{array}{l}\text { eng begrenzt } \\
\text { Kategorien 7-9 }\end{array}$ \\
$\begin{array}{l}\text { relevante Räume/ } \\
\text { induationen }\end{array}$ & $\begin{array}{l}\text { auf Räume } \\
\text { keine lokalisierten Körperpositionen }\end{array}$ & $\begin{array}{l}\text { indexikalisch oder absolut be- } \\
\text { stimmter Raum } \\
\text { bestimmte lokalisierte Körperposi- } \\
\text { tionen }\end{array}$ \\
\hline
\end{tabular}


>Abstand halten< ein Sich-Hinstellen auf den Aufkleber selbst, also das Einnehmen einer Körperposition, das synchron mit dem Lesen einhergeht. Dies ist bei den anderen Kommunikaten in der Regel nicht der Fall, die Positionierung bei der Rezeption kann allenfalls erschlossen werden dadurch, dass eine minimale und maximale Entfernung vom zu lesenden Kommunikat vorausgesetzt wird - man muss sie maximal (noch) lesen können, und man hält allgemein einen gewissen minimalen Abstand zu ihnen ein. Ebenfalls inferiert wird beispielsweise die durchschnittliche Augenhöhe der Lesenden, was einen Einfluss auf die Höhe der Anbringung hat.

In der folgenden Klassifikation werden innerhalb der drei Kategorien der allgemeinen, weiten und engen Reichweite jeweils drei Funktionen unterschieden, die oben schon eingeführt wurden: die Funktion der Expressivität, der Regulierung und der Information. Auf der unteren Granulationsebene liegen also neun Kategorien vor, die im Folgenden näher erläutert und beschrieben werden sollen.

\subsection{Corona-Kommunikate mit allgemeiner Geltung}

Kategorie 1. Allgemein/Expressiv: Hier finden sich vor allem allgemeine Bekundungen von Dank oder Solidarität, aber auch Formen von Forderung oder Protest (s. Abb. 14). Der Anbringungsort hat keinen oder nur einen geringen Einfluss auf die Reichweite bzw. den Geltungsbereich der Botschaft, denn sie ist lokal nicht spezifiziert. Sie wird im Vorbeigehen oder -fahren rezipiert, weshalb ihre Träger typischerweise an Orten mit hoher Verkehrsdichte angebracht sind. Dies sind Straßen, Haltestellen und Ähnliches. Auch die Größe und Farbe der Schrift oder anderer
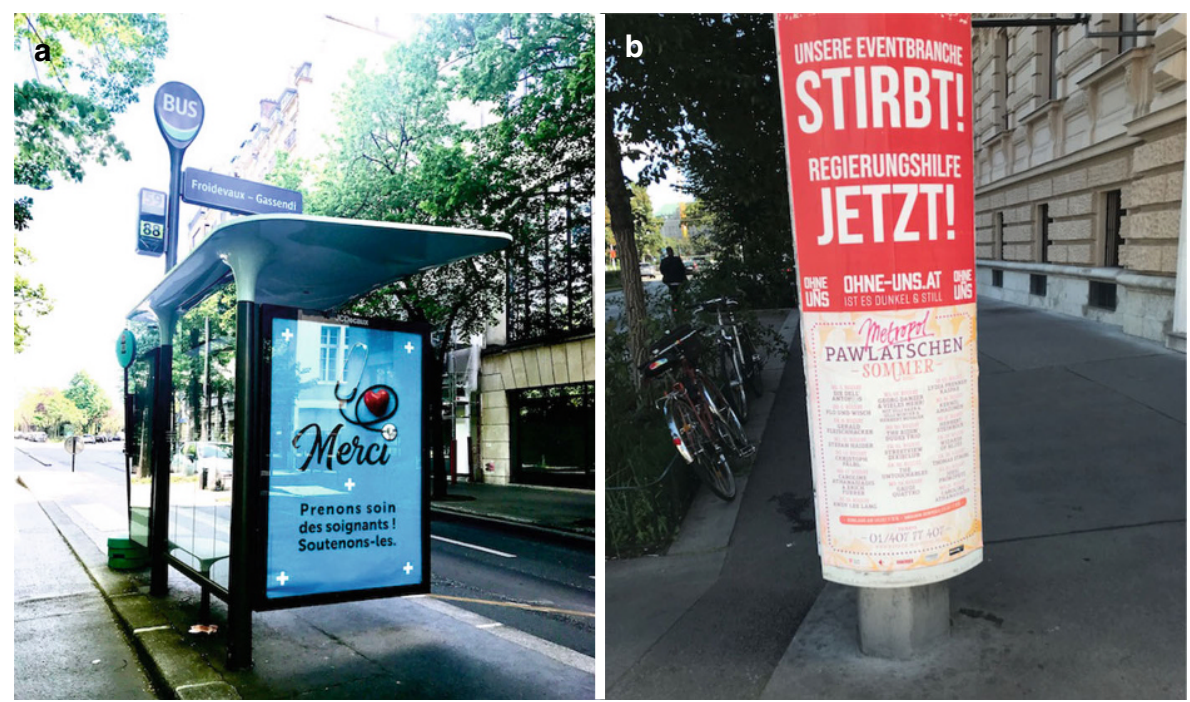

Abb. 14 Emotionale Botschaften oder Protestbekundungen mit allgemeiner Reichweite (Kategorie 1. Allgemein/Expressiv). a Paris, Frühjahr 2020, Plakat an einer Bushaltestelle: >Danke. Achten wir auf die Pflegekräfte. Unterstützen wir sie! < b Wien, Sommer 2020, Straßenplakat der Vereinigung der österreichischen Veranstaltungsdienstleister: Unsere Eventbranche STIRBT! Regierungshilfe JETZT! 


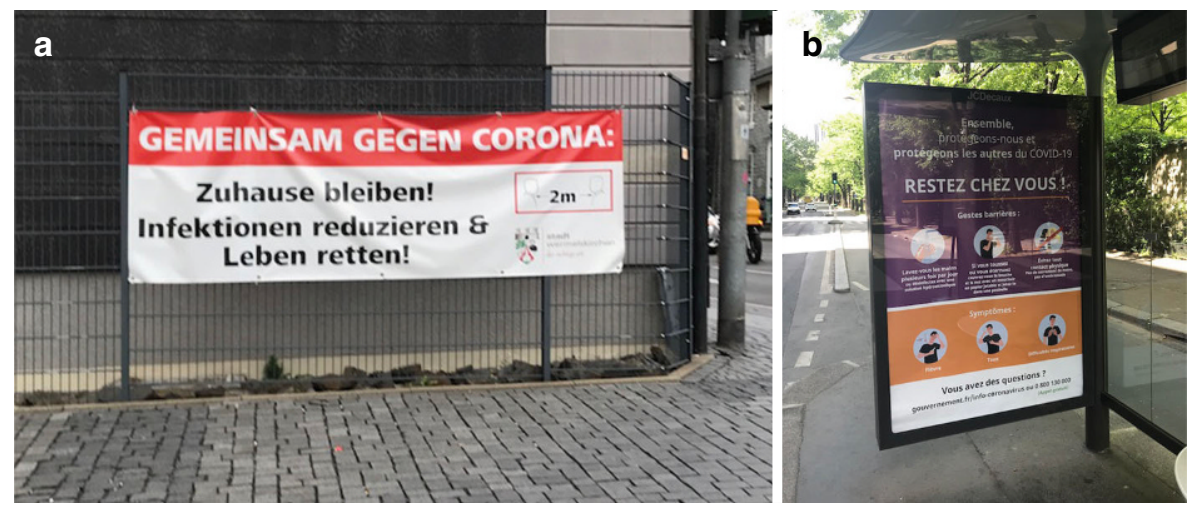

Abb. 15 Regulierende Botschaften (Kategorie 2. Allgemein/Regulierend). a Wermelskirchen, 2020: GEMEINSAM GEGEN CORONA Zuhause bleiben! Infektionen reduzieren \& Leben retten, b Paris, Frühjahr 2020: >Gemeinsam, schützen wir uns und die anderen. Bleibt/Bleiben Sie zu Hause!<

Abb. 16 Umweltverschmutzung durch MNS (Kategorie 3. Allgemein/Informativ). Städtische Kommunikationstafel, Nizza, Sommer 2020: >Er rettet Leben auf dem Festland, aber er tötet im Meer< $(\mathrm{er}=$ der MundNasen-Schutz)

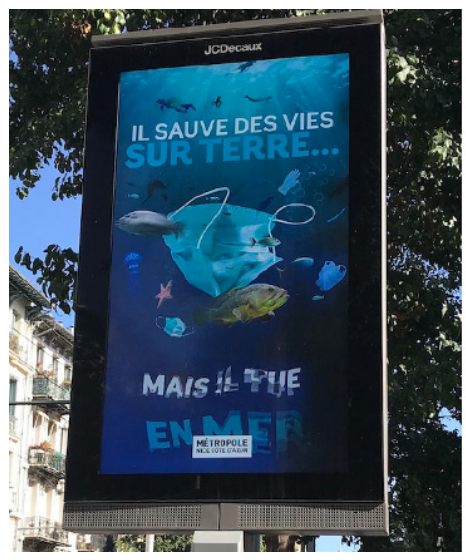

Zeichen ist der transitorischen Rezeptionssituation angepasst, sie sind deutlich und schnell erkennbar.

Kategorie 2. Allgemein/Regulierend: Auch hier ist der Anbringungsort Teil eines kommunikativen Dispositivs (Stadt, Bahnhof etc.), die Botschaft ist eine allgemeingültige Aufforderung - sie richtet sich an jeden (Abb. 15). Entsprechend sind diese Kommunikate ebenfalls an Orten mit hoher Verkehrsdichte angebracht, an denen die Chancen der Rezeption groß sind. Im deutschen Beispiel ist der durchgängige Slogan »Gemeinsam gegen Corona « den drei Appellen vorgeschaltet. Im französischen Beispiel wird ebenfalls die Gemeinsamkeit der erforderlichen Handlungsweisen betont: »Ensemble, protégeons-nous et protégeons les autres« >Gemeinsam, schützen wir uns und die anderen $<$.

Kategorie 3. Allgemein/Informativ: Das Kommunikat ist qua Verfasser*in und Anbringungsort Teil eines städtischen oder institutionellen Dispositivs, es handelt sich v.a. um Informationen zur Pandemie. Eine dafür vorgesehene beleuchtete Anzeigetafel, die für wechselnde Aushänge geeignet ist, informiert über die Folgen des 
achtlosen Wegwerfens des Mund-Nasen-Schutzes (Abb. 16). Das Gegensatzpaar Leben/Tod wird hier rhetorisch eingesetzt.

\subsection{Corona-Kommunikate mit areal weit spezifizierter Geltung}

Die Gesamtsituation im Falle einer arealen Spezifizierung, die den Aushang oder den Aufkleber und die verschiedenen Elemente der Lektüresituation sowie des -kontextes umfasst, besteht in einem dichten Beziehungsnetz, das Semiotisches, Materielles und Körperliches umfasst, wie wir es mit Jannis Androutsopoulos (2020) schon festgestellt hatten. In den folgenden beiden Beispielen ist die Lektüresituation durch die Institution des Flughafens gekennzeichnet, der einen transitorischen Ort (nach Marc Augé 1992 wohl einen >Nicht-Ort<) für einen anschließenden Aufenthalt im Gültigkeitsbereich der Botschaft darstellt.

Kategorie 4. Weit/Expressiv: Eine Aufschrift wie »Paris vous n'oublira pas/Paris will never forget you « >Paris vergisst Dich/Sie nicht< in der Abflughalle eines Flughafens ist für eine Situation geprägt, die mit dem Abflug der Reisenden (= Extension von vous/you) eng zusammenhängt (Abb. 17b). Die Nennung des Stadtnamens erhält durch das Abreisen der Rezipient*innen aus der Stadt ihre Relevanz, der Gehalt des kommissiven Sprechakts - der gleichzeitig einen Trost oder eine Ermutigung beinhaltet - bezieht sich implizit auf die folgende Reise. Der Wunsch »Bleiben Sie gesund/Stay safe « im Abflugsbereich des Wiener Flughafens (Abb. 17a) (als Emittent ist Vienna International Airport genannt), bezieht sich ebenfalls auf die Fluggäste und ihre anschließende Reise.

Kategorie 5. Weit/Regulierend: Der Anbringungsort der regulativen Botschaft bestimmt einen Gültigkeitsraum, der entweder explizit genannt oder aufgrund der lokalen Gegebenheiten erschlossen wird. »Dans ce secteur« >in diesem Sektor/Bereich bezieht sich auf den Teil eines Ortes, innerhalb dessen das Maskentragen obligatorisch ist (Abb. 18b). Die Tafel fungiert als Grenzmarkierung, die ein >obligatorisches< (in der Markthalle) und ein >freies « Areal (außerhalb) voneinander abgrenzt. Die Aufforderung, eine Gruppenbildung zu unterlassen, bezieht sich auf das Waldstück,
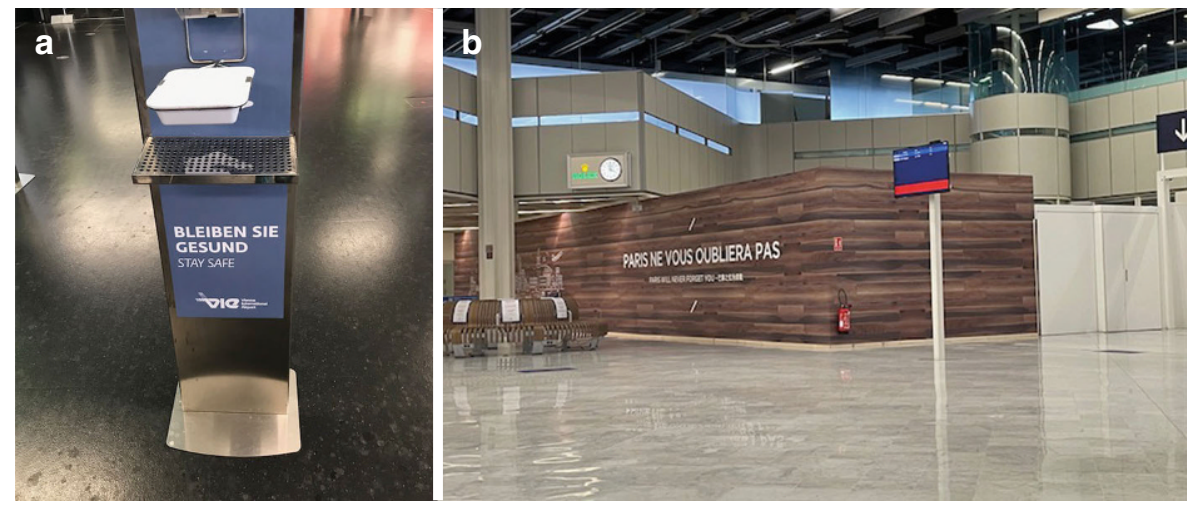

Abb. 17 Expressive Kommunikate in Flughäfen (Kategorie 4. Weit/Expressiv). a Flughafen Wien, Sommer 2020: BLEIBEN SIE GESUnd STAY SAFE, b Flughafen Orly, Paris, Februar 2021: >Paris vergisst Dich/Sie nicht< 

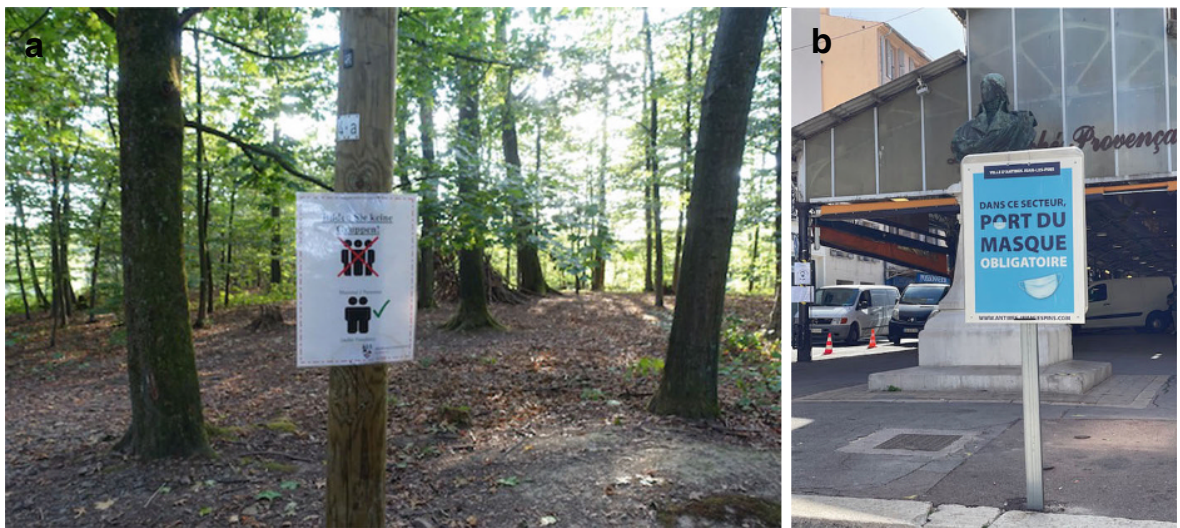

Abb. 18 Vorschriften, ein relativ weiten Bereich betreffend (Kategorie 5. Weit/Regulierend). a Wermelskirchen, Sommer 2020, im Wald neben einem Friedhof: BILDEN SIE KEINE GRUPPEN, b Antibes, Februar 2021: >In diesem Sektor / Maskentragen / Obligatoirsich<
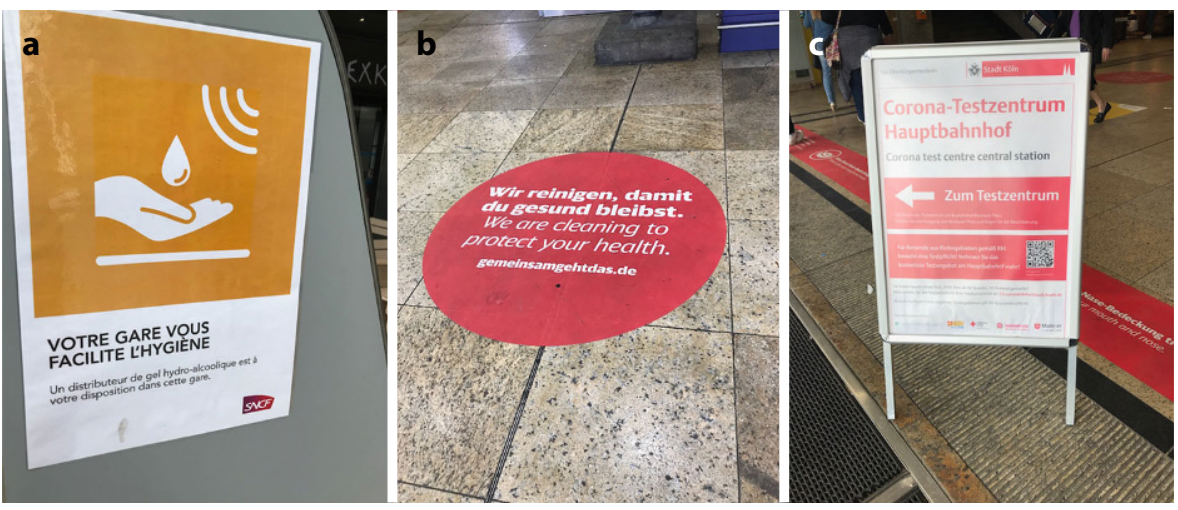

Abb. 19 Informationen zu Desinfektionsmöglichkeiten/vermehrtem Reinigen, Lokalisierung eines Testzentrums (Kategorie 6. Weit/Informativ). a Gare Montparnasse, Paris, 2020: >Hygiene im Bahnhof leicht gemacht. Ein Spender mit hydro-alkoholischem Gel steht Ihnen in diesem Bahnhof zur Verfügung<, b Hauptbahnhof Köln, Sommer 2020: Wir reinigen, damit du gesund bleibst. gemeinsamgehtdas.de, c Hauptbahnhof Köln, Sommer 2020: Corona-Testzentrum Hauptbahnhof [Pfeil nach links] Zum Testzentrum

in dem der Aushang angebracht ist (Abb. 18a). Das Verbot gilt auch anderswo, die Relevanz des Schildes ist durch die Tendenz gegeben, dass sich Waldspaziergänger oft in kleineren Gruppen bewegen.

Kategorie 6. Weit/Informativ: Das Kommunikat ist Teil eines kommunikativen Dispositivs auf der Ebene der Meso-Kommunikation. Die Botschaft betrifft die angebotenen Corona-Vorrichtungen und Handlungen der betreffenden Institution. So wird auf die Möglichkeiten des Hygieneverhaltens hingewiesen, oder es wird vermehrtes Reinigen damit begründet, dass man die Gesundheit der Besucher*innen schütze. Ein Verweis auf den Ort erfolgt entweder explizit (»dans cette gare «, >in diesem Bahnhof<, Abb. 19a) oder dieser wird implizit vorausgesetzt (Abb. 19b). Der 
Slogan in Form einer URL (gemeinsamgehtdas.de) ist als Appell an das kollektive Verhalten zu werten und unterstützt die jeweilige Botschaft. Die Kommunikate können auch mittels eines Pfeils auf bestimmte Orte innerhalb des Areals verweisen, um z.B. Testzentren anzuzeigen (Abb. 19c).

\subsection{Corona-Kommunikate mit areal eng spezifizierter Geltung}

Kommunikate mit areal enger Reichweite wirken - vor allem in ihrer regulierenden Funktion - auf die körperliche Positionierung oder ihre Unterlassung durch die Lesenden ein, entweder in Form von Geboten oder von Sperren, z.B. eines Sitzplatzes. Die entsprechenden Markierungen haben teilweise ikonischen Charakter, indem sie die Fußform der Stehenden abbilden, oder sie erscheinen als einfache Linien, die aufgeklebt oder aufgesprüht sind und so Markierungen für einzuhaltende Abstände darstellen (s.u. Abb. 22a). Das geforderte Verhalten wird dabei synchron zur Lektüre ausgeführt.

Diese Klasse von Kommunikaten bildet ein »soziomaterielles Dispositiv« (Habscheid/Reuther 2013), um ein bestimmtes, örtlich eng begrenztes Verhalten einzufordern bzw. zu erzwingen. Die Kommunikate mit ihrer materiellen und sprachlichen Komponente haben hier präzise performative Macht (Peeters/Charlier 1999) bzw. erzwingen Faktizität (Habscheid/Reuther 2013, Felder 2013). M. a. W., sie greifen an bestimmten Punkten sehr stark und direkt in das Handeln und Verhalten der Personen ein - im Gegensatz zu den regulativen Kommunikaten mit weiter oder allgemeiner Reichweite, deren Befolgung nicht synchron zur Lektüre erfolgt und leichter umgangen werden kann.

Kategorie 7. Eng/Expressiv: Der Anbringungsort ist Exponent eines Emotionsträgers - z.B. eine Vitrine. Die Botschaft (Abb. 20) bezieht sich auf die Stadtflucht der Pariser*innen nach der Ankündigung des dritten Lockdowns: >Städtischer Exodus des Schaufensters $<$. Die Auslagen sind dementsprechend ausgedünnt.

Kategorie 8. Eng/Regulierend: Der Anbringungsort bestimmt einen sehr engen Gültigkeitsraum, in dem ein bestimmtes Verhalten gefordert ist. In den Beispielen Abb. 21 wird das Platznehmen untersagt, wobei die konkrete Sitzgelegenheit gemeint

Abb. 20 Metareflexiver Aushang, Paris, Frühjahr 2021, Auslage eines Bierspezialitätengeschäfts $>$ Städtischer Exodus I des I Schaufensters

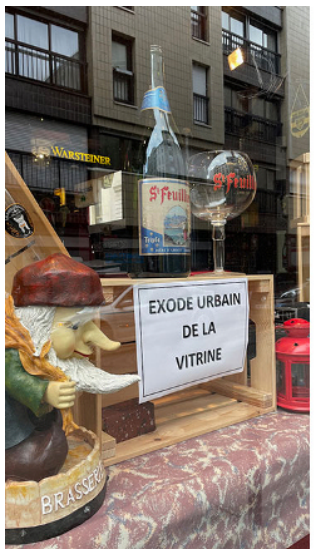



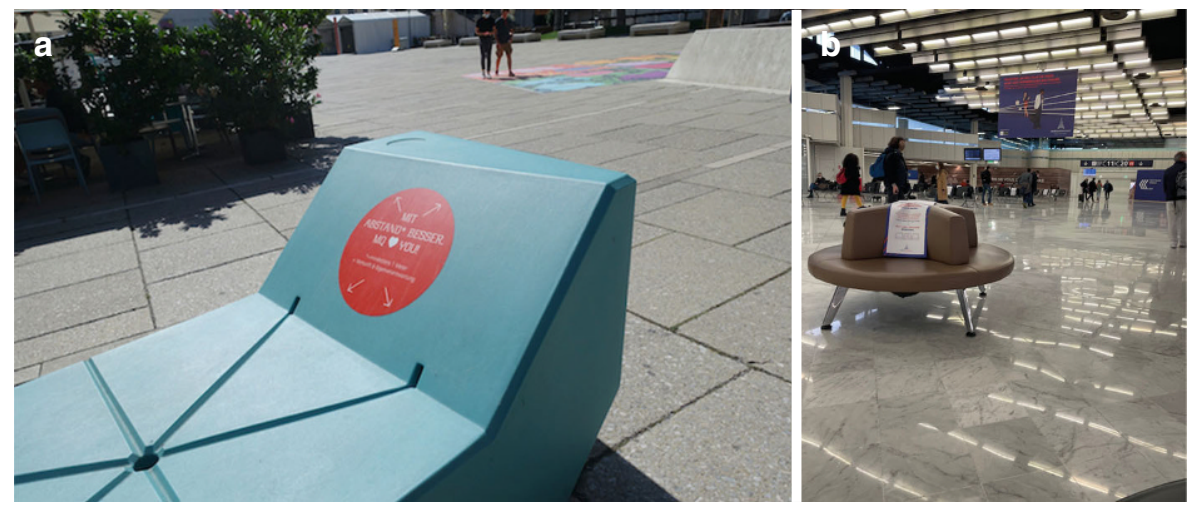

Abb. 21 Platznehmen in Zeiten des Corona-Virus (Kategorie 8. Eng/Regulierend). a Wien, Sommer 2020: MIT ABSTAND BeSSER. MQ [HeRZ] YOU! b Flughafen Paris, Februar 2021: >Sitz freilassen<
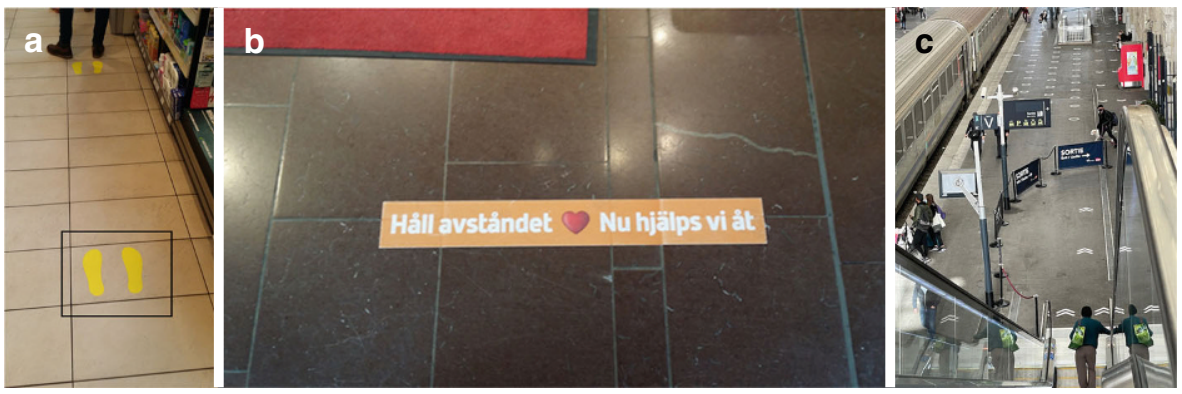

Abb. 22 Strikte Regulierung der aufrechten statischen bzw. dynamischen Positionierung (Kategorie 8. Eng/Regulierend). a Düsseldorf, Sommer 2020, Kassenbereich einer Tankstelle: [Fußabdrücke], b Göteborg, Sommer 2020, Aufforderung zum Abstandhalten im Bahnhof: >Haltet Abstand [Herzsymbol] so ist uns allen geholfen<, c Nizza, Februar 2021, Regulierung des Publikumsverkehrs im Bahnhof: [Bodenmarkierung] >Ausgang< [auf den Absperrungen]

ist, die markiert wurde. In den Beispielen Abb. 22 wiederum wird ein konkretes Verhalten entweder statisch (Sich-Hinstellen) oder dynamisch (in eine bestimmte Richtung gehen) vorgeschrieben.

Kategorie 9. Eng/Informativ: Der dingfeste (Bühler 1934/1978, § 10, 2) Anbringungsort des informativen Kommunikats prädiziert Eigenschaften oder identifiziert die Funktion eines bestimmten Ortes/Objekts. Ein auf dem Tisch aufgeklebter QRCode, hier in Wien, gibt Zugang zur Speisekarte eines Restaurants, ohne Manipulierung der Karte durch den Gast oder die Bedienung (Abb. 23a). Neben einem Wasserund Seifenspender in einem Pariser Park wurde ein spezieller DesinfektionsmittelSpender aufgestellt (Abb. 23b). 

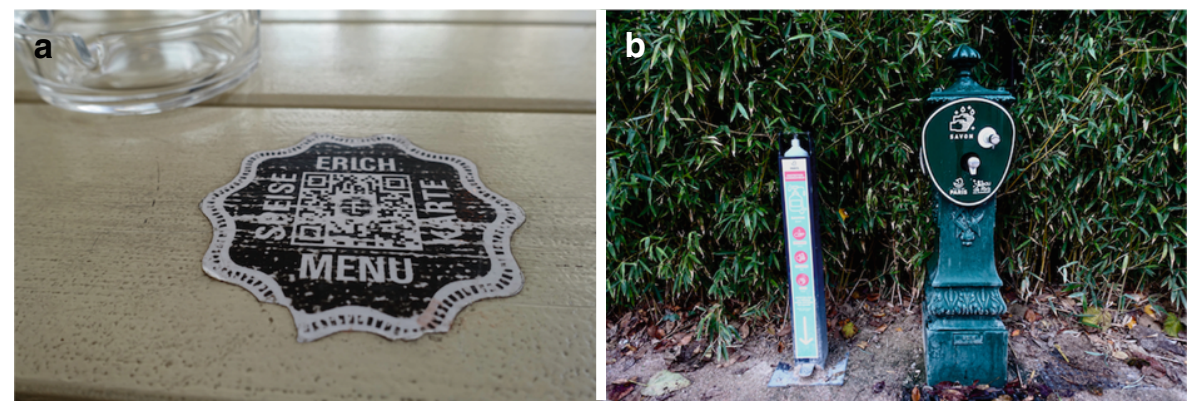

Abb. 23 Dingfest angebrachte Informationen (Kategorie 9. Eng/Informativ). a Wien Sommer 2020, aufgeklebter QR-Code gibt Zugang zur Speisekarte, b Paris 2020, Spender mit Fußpedal für Desinfektionsmittel in einem Park: >Corona-Schutz<

\section{Schlussbetrachtungen}

Können wir am Ende dieser Überlegungen eine Antwort auf die Frage geben, wie die kleinformatigen Corona-Kommunikate im öffentlichen Raum die Gesundheitskrise mit-konstituieren? Wir meinen, Folgendes sagen zu können:

a) Die Corona-Kommunikate funktionieren auf dem mehrfachen Hintergrund des allgemeinen öffentlichen Corona-Diskurses, den sie lokal transkribieren.

b) Sie richten sich an Einzelpersonen und Gruppen, die zumeist zu Fuß unterwegs sind, verschiedenen Aktivitäten nachgehen und dabei die Kommunikate wahrnehmen.

c) Die Corona-Kommunikate bedienen expressive, regulative und informative Bedürfnisse verschiedener - nämlich öffentlicher, kommenzieller und privater - Autoren, die nicht-routinehafte Situationen thematisieren. Bei gleicher funktioneller Ausrichtung haben die Autor*innen ab einem bestimmten Zeitpunkt der Krise die Wahl zwischen formularhaften und frei formulierten Formaten, die auch kombiniert werden können.

d) Im Laufe der Monate von März 2020 bis März 2021 können wir Entwicklungen feststellen. Wenn die drei Hauptfunktionen (Expressivität, Regulation, Information) in allen Phasen der Pandemie vertreten sind, so gibt es Unterschiede in der Kreativität und in der Präponderanz verschiedener Funktionen in bestimmten Phasen. Konkret bedeutet das, dass die erste und die zweite Phase sehr kreative Phasen sind, in der die verschiedenen neuen Kommunikate erfunden werden und in der die expressive Funktion am stärksten vertreten ist. Die dritte Phase, die der chronischen Krise hingegen, ist eine Phase der Adaptation an ständig wechselnde Vorschriften verschiedener Art.

e) Die Kommunikate haben unterschiedliche Reichweiten und Geltungsbereiche, sie tangieren die Rezipient*innen in verschiedener Weise - die Spannweite reicht von ganz allgemeinen Botschaften bis zu konkreten, punktuellen Einschränkungen der Bewegungsfreiheit. Diese Spannbreite der Arealität kennzeichnet alle Phasen, sowohl öffentliche als auch kommerzielle und private Bereiche, sowie die Diskursfunktionen (Expressivität, Regulation, Information). Es gibt also ein multifakto- 
rielles Kontinuum von allgemeinen expressiven Botschaften, die die Lesenden vielleicht nur emotional berühren, bis zu räumlich präzisen, einschränkenden Vorschriften, die den Lesenden konkrete Handlungen und Verhaltensweisen, ja sogar Körperhaltungen vorschreiben.

Indem sie die Verhaltens- und Handlungsaspekte der öffentlichen Anti-CoronaVorschriften transkribieren, sind die Corona-Kommunikate Teil der Krise im Sinne der Konstitution von neuen Routinen. Indem sie Raum für verschiedene Arten der Expressivität geben, sind sie aber auch Teil der emotionalen Bewältigung der Krise in dem Sinne, dass sie Ausdrucksmöglichkeiten für Emotionen und Appelle schaffen, sowie Solidarität benennen und einfordern. Darin liegt die Besonderheit der CoronaKommunikate im Vergleich zu anderen Bereichen der öffentlichen Kommunikation über kleinformatige sprachliche und bild-sprachliche Kommunikate. Nicht zuletzt ergibt sich durch die teilweise dichte Präsenz, die Kreativität der Verfasser und die verschiedenen Reichweiten eine bewegliche, bunte Sprachlandschaft.

Abschließend kann gesagt werden, dass die Hypothese der Arealität als einer auch klassifikatorisch entscheidenden Eigenschaft der untersuchten Kommunikate vor dem Hintergrund des untersuchten Materials als vorläufig bestätigt gelten kann. Die Annahme der Konstitution der Krise durch die Kommunikate wiederum sollte angesichts der engen Verschränkung von Krise und ihrer Kommunikation ebenfalls an dem Material augenfällig geworden sein.

\section{Literatur}

\section{Verwendete Literatur}

Androutsopoulos, Jannis (2020): Die Sprachlandschaft im Dispositiv der Pandemie. In: Aptum. Zeitschrift für Sprachkritik und Sprachkultur. 16. Jahrgang, 2020, Heft 02/03, S. 290-299.

Auer, Peter (2010): Sprachliche Landschaften. In: Arnulf Deppermann/Angelika Linke (Hg.). Sprache intermedial. Stimme und Schrift, Bild und Ton. Berlin/New York: De Gruyter, S. 271-298 (= Institut für deutsche Sprache, Jahrbuch 2009).

Augé, Marc (1992): Non-lieux. Introduction à une anthropologie de la surmodernité. Paris: Seuil.

Behr, Irmtraud (2005): Petite stylistique des panneaux >régulateurs<. In: Irmtraud Behr/Peter Henning (Hg.). À travers champs. Mélanges en l'honneur de Nicole Fernandez- Bravo. Paris: L'Harmattan, S. 333-347.

Behr, Irmtraud (2019): Indexicalité linguistique, iconique et matérielle - l'emploi du déictique »hier« dans quelques panneaux allemands. In: Faits de Langue 49.2, S. 41-54.

Bosch, Aida (2015): Unsicherheit, Krise und Routine. Zur Rolle der Dinge in der menschlichen Lebenswelt. In: Christoph Wulf/Jörg Zifas (Hg.): Unsicherheit. Paragrana, Internationale Zeitschrift für Historische Anthropologie Bd. 24/2015/H1, Berlin/Boston/Peking: De Gruyter, S. 209-220.

Bühler, Karl (1934/1978): Sprachtheorie. Frankfurt am Main/Berlin/Wien: Ullstein.

Domke, Christin (2013): Ortsgebundenheit als distinktives Merkmal in der Textanalyse. In: ZGL 2013; 41(1), S. 102-126.

Dreesen, Philipp/Pohl, Peter C. (2020): Chronifizierung der Krise. Ein, zugegeben gedrängter, Versuch zum Zeitlichkeitsdiskurs von Corona. In: Aptum. Zeitschrift für Sprachkritik und Sprachkultur. 16. Jahrgang, 2020, Heft 02/03, S. 274-280.

Felder, Ekkehard (2013): Faktizitätsherstellung mittels handlungsleitender Konzepte und agonaler Zentren. Der diskursive Wettkampf um Geltungsansprüche. In: Ekkehard Felder (Hg.): Faktizitätsherstellung in Diskursen. Die Macht des Deklarativen. Berlin/New York: De Gruyter, S. 13-28 (= Sprache und Wissen 13). 
Habscheid, Stephan/Reuther, Nadine (2013): Performatisierung und Verräumlichung von Diskursen. Zur soziomateriellen Herstellung von >Sicherheit< an öffentlichen Orten. In: Ekkehard Felder (Hg.): Faktizitätsherstellung in Diskursen. Die Macht des Deklarativen. Berlin/New York: De Gruyter, S. 127-145 (= Sprache und Wissen 13).

Hausendorf, Heiko (2009): Kleine Texte - über Randerscheinungen von Textualität. In: Germanistik in der Schweiz - Onlinezeitschrift der Schweizerischen Akademischen Gesellschaft für Germanistik 6, S. 5-19.

Hennig, Mathilde (2010): Grammatik multikodal: ein Vorschlag am Beispiel ortsgebundener Schriftlichkeit. In: Kodikas/Code. Ars Semiotica 33/1-2, S. 73-88.

Jäger, Ludwig (2010): Intermedialität - Intramedialität - Transkriptivität. Überlegungen zu einigen Prinzipien der kulturellen Semiosis. In: Arnulf Deppermann/Angelika Linke (Hg.): Sprache intermedial. Stimme und Schrift, Bild und Ton. Berlin/New York: de Gruyter, 2010. S. 301-323. (Jahrbuch des Instituts für Deutsche Sprache 2009). https://doi.org/10.1515/9783110223613.299

Kant, Immanuel (1974): Kritik der reinen Vernunft. Frankfurt/M.: Suhrkamp. (A 1781; B 1787)

Kavanagh, Éric/Roberge, Jacynthe/Sperano, Isabelle (2016): Typologie exploratoire des affordances textuelles. In: Pratiques [En ligne], S. 171-172. URL: http://journals.openedition.org/pratiques/3187. https://doi.org/10.4000/pratiques.3187 (4.4.2021)

Liedtke, Frank (2020): Wirus oder: Was es heißt, solidarisch zu sein. In: Aptum. Zeitschrift für Sprachkritik und Sprachkultur 16/2020, 2/3, S. 134-141.

Mead, George Herbert (1938): Philosophy of the Present. Chicago: Open Court.

Oevermann, Ulrich (2006): Wissen, Glauben, Überzeugung. Ein Vorschlag zu einer Theorie des Wissens aus krisentheoretischer Perspektive. In: D. Tänzler/H. Knoblauch/H.-G. Soeffner (Hg.): Neue Perspektiven der Wissenssoziologie. Konstanz: UVK Verlagsgesellschaft, S. 79-118.

Parr, Rolf (2013): Krise/Katastrophe. Normalismustheoretische Überlegungen zu einem semantischen Diferential. In: Martin Wengeler/Alexander Ziem (Hg.): Sprachliche Konstruktionen von Krise. Interdisziplinäre Perspektiven auf ein fortwährend aktuelles Phänomen. Bremen: Hempen, S. 289-303.

Peeters, Hugues/Charlier, Philippe (Hg.) (1999): Contributions à une théorie du dispositif. In: Hermès, La Revue, $1999 / 3$ n $^{\circ} 25$, S. 15-23.

Polenz, Peter von (1988): Deutsche Satzsemantik. Berlin/New York: De Gruyter.

Römer, David (2020): Krisendiskurs. In: Thomas Niehr/Jörg Kilian/Jochen Schiewe (Hg.), Handbuch Sprachkritik, Stuttgart: Metzler, S. 233-240.

Schmitz, Ulrich (2010): Sehflächenforschung. Eine Einführung. In: http://www.linse.uni-due.de/files/ PDF_in_Katalog/publikationen/Online-Publikationen/Sehflchenforschung-2010.pdf. (4.4.2021)

Schütz, Alfred/Luckmann, Thomas (1979): Strukturen der Lebenswelt. Frankfurt/M.: Suhrkamp.

Scollon, Ron/Scollon, Suzie Wong (2003): Discourses in Place. Language in the Material World. London: Routledge.

Spieß, Constanze (2020): »Passen Sie gut auf sich und Ihre Liebsten auf« und »Vive la France!« - Linguistische Anmerkungen zu den TV-Ansprachen von Merkel und Macron. In: Aptum. Zeitschrift für Sprachkritik und Sprachkultur 16/2020, 2/3, S. 206-211.

Wengeler, Martin/Ziem, Alexander (2010): »Wirtschaftskrisen« im Wandel der Zeit. Eine diskurslinguistische Pilotstudie zum Wandel von Argumentationsmustern und Metapherngebrauch. In: Achim Landwehr (Hg.): Diskursiver Wandel. Wiesbaden, S. 335-354.

\section{Internetseiten}

Gensburger, Sarah/Severo, Marta (2020): Défi collaboratif VintrinesEnConfinement. In: https:// vitrinesenconfinement.huma-num.fr/ (4.4.2021).

GfDS: https://gfds.de/wort-des-jahres-2020-1 (4.4.2021).

gouverment.fr: https://www.gouvernement.fr/risques/comprendre-le-plan-vigipirate (4.4.2021).

oesterreich.gv.at: https://www.oesterreich.gv.at (4.4.2021).

santepubliquefrance.fr: https://www.santepubliquefrance.fr/maladies-et-traumatismes/maladies-et-infec tions-respiratoires/infection-a-coronavirus/documents/depliant-flyer/covid-19-supports-d-informationgrand-public.-catalogue-janvier-2021 (4.4.2021) 